\title{
HIFs, angiogenesis, and metabolism: elusive enemies in breast cancer
}

\author{
Ellen C. de Heer, ${ }^{1}$ Mathilde Jalving, ${ }^{1}$ and Adrian L. Harris ${ }^{2}$ \\ 'University of Groningen, University Medical Center Groningen, Department of Medical Oncology, Groningen, Netherlands. ${ }^{2}$ Molecular Oncology Laboratories, Weatherall Institute of Molecular Medicine, \\ University of Oxford, Oxford, United Kingdom.
}

\begin{abstract}
Hypoxia-inducible factors (HIFs) and the HIF-dependent cancer hallmarks angiogenesis and metabolic rewiring are well-established drivers of breast cancer aggressiveness, therapy resistance, and poor prognosis. Targeting of HIF and its downstream targets in angiogenesis and metabolism has been unsuccessful so far in the breast cancer clinical setting, with major unresolved challenges residing in target selection, development of robust biomarkers for response prediction, and understanding and harnessing of escape mechanisms. This Review discusses the pathophysiological role of HIFs, angiogenesis, and metabolism in breast cancer and the challenges of targeting these features in patients with breast cancer. Rational therapeutic combinations, especially with immunotherapy and endocrine therapy, seem most promising in the clinical exploitation of the intricate interplay of HIFs, angiogenesis, and metabolism in breast cancer cells and the tumor microenvironment.
\end{abstract}

\section{Introduction}

Breast cancer is the cancer type with the highest prevalence and, despite therapeutic advances, still has the second highest cancer-related mortality rate in women (1). In breast cancer, low intratumoral $\mathrm{O}_{2}$ levels (hypoxia) are associated with aggressive tumor behavior, metastasis, and resistance to therapy. The first in vivo measurements of oxygen content and subsequent observation of hypoxia in patients' breast tumors were described nearly 30 years ago (2). The transcription factor hypoxia-inducible factor 1 (HIF-1) was later characterized as the master regulator of cellular adaptation to hypoxia (3). The vital role of HIFs in every hallmark of cancer, in tumor progression, and in therapy resistance is now well established (4). Two fundamental processes that are especially dependent on HIFs are metabolic rewiring resulting in a more oxygen-independent nutrient metabolism, and angiogenesis, i.e., the growth of new blood vessels from preexisting vasculature. Targeting of key players in metabolic and angiogenic pathways in breast cancer has yielded disappointing results, the most notable being the lack of overall survival benefit of the antiangiogenic agent bevacizumab, which targets VEGF (5). This Review provides an overview of HIF-dependent reprogramming of angiogenic and metabolic pathways in breast cancer and discusses novel approaches and challenges in the clinical translation of this knowledge into successful treatment strategies.

Conflict of interest: ALH serves in a paid advisory role for Curve Therapeutics MJ serves in an advisory role for Bristol Myers Squibb, Merck \& Co., Novartis, AstraZeneca, Tesaro, and Pierre Fabre (fees paid to the institution).

Copyright: () 2020, American Society for Clinical Investigation.

Reference information: J Clin Invest. 2020;130(10):5074-5087. https://doi.org/10.1172/JCI137552.

\section{HIF activity in breast cancer}

Active HIF is composed of the constitutively expressed HIF-1 $\beta$ subunit, an $\mathrm{O}_{2}$-dependent $\mathrm{HIF} \alpha$ isoform, and essential cofactors. HIF induces transcription of target genes by binding to hypoxia-responsive elements (HREs) in promoters. As in all mammalian cells, in breast cancer, HIF $\alpha$ stability and corresponding HIF activity are greatly increased in hypoxia (Figure 1). In normoxia, HIF activity is repressed through proteasomal degradation of $\mathrm{HIF} \alpha$ by the $\mathrm{O}_{2}$-dependent prolyl hydroxylase domain (PHD) proteins and the von Hippel-Lindau (VHL) protein, and/or by inhibition of HIF $\alpha$ binding to essential cofactors by factor inhibiting HIF-1 (FIH-1) (6). Downstream targets of the HIF $\alpha$ isoforms (HIF$1 \alpha$ and HIF- $2 \alpha$ ) only partially overlap, and in breast cancer, HIF$1 \alpha$ is the predominantly (over)expressed isoform $(7,8)$. Recently, specific roles for HIF-2 $\alpha$ in breast cancer progression, mediated upstream by the transcription factor FOXA1, and in angiogenesis have been identified $(9,10)$. In human breast tumors, HIF-1 $\alpha$ is already overexpressed in precursor lesions (ductal carcinoma in situ [DCIS]) and early-stage breast cancer, and these levels strongly correlate with tumor grade and invasion (11). HIF- $1 \alpha$ foci are predominantly observed surrounding necrotic areas such as the generally hypoxic tumor core.

Common genetic alterations in breast cancer, such as loss of the tumor suppressors PTEN, p53, or BRCA1 and hyperactivation of the $\mathrm{PI} 3 \mathrm{~K} / \mathrm{Akt} / \mathrm{mTOR}$ or MAPK pathway, increase HIF $\alpha$ transcription, translation, or stability independently of $\mathrm{O}_{2}$ levels (refs. 4, 12, 13, and Figure 1). Human epidermal growth factor receptor 2 (HER2; overexpressed in $15 \%-30 \%$ of human breast cancers) and estrogen receptor- $\alpha$ (ER $\alpha$; positive in approximately $70 \%$ of breast cancers) increase HIF $\alpha$ levels through increased PI3K/Akt/mTOR signaling $(14,15)$. $\mathrm{ER} \alpha$ also directly induces HIF- $1 \alpha$, but not HIF- $2 \alpha$, expression through an estrogen response element in the $\operatorname{HIF} 1 A$ promoter $(16,17)$. 


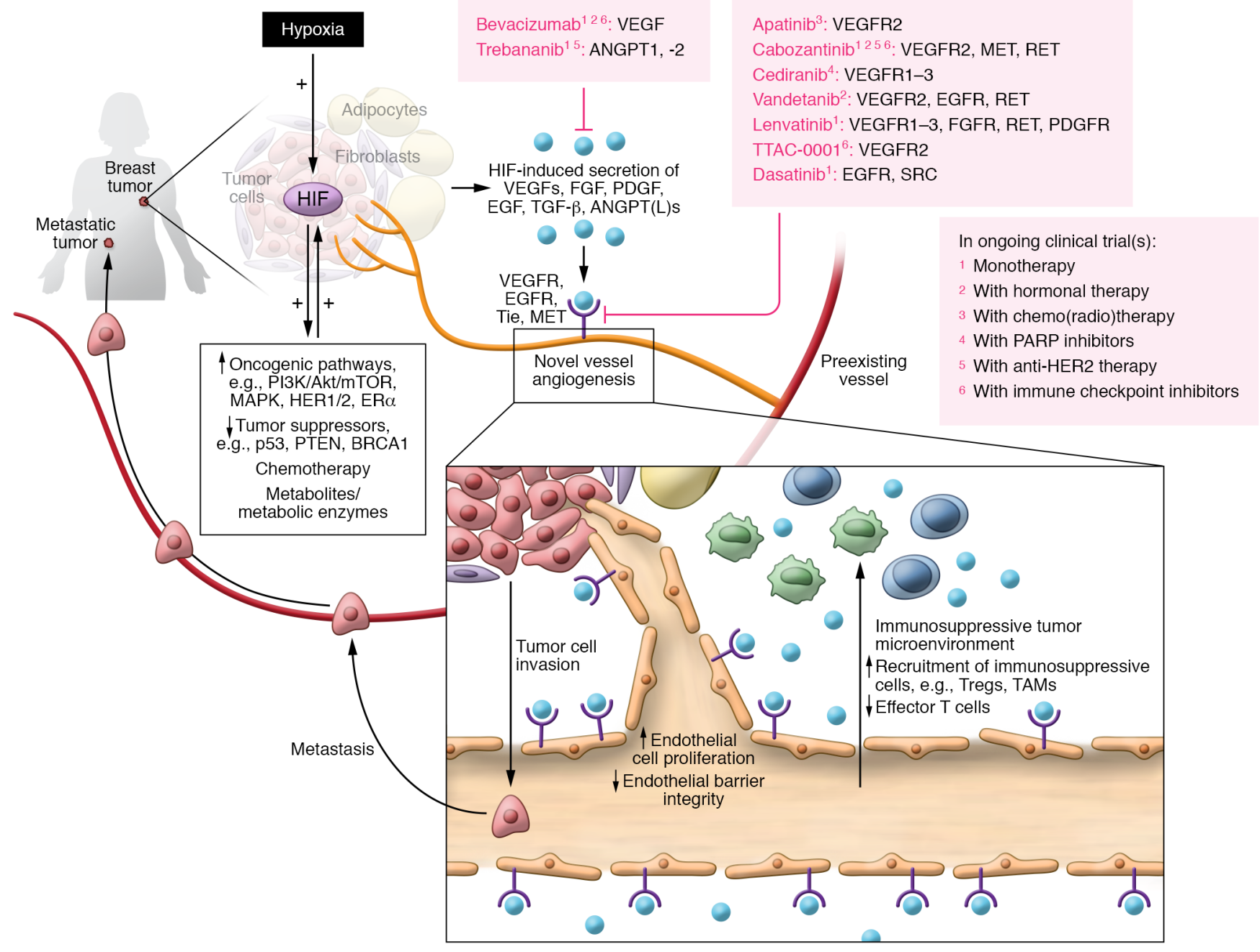

Figure 1. Schematic overview of HIFs and HIF-induced angiogenesis in breast cancer. HIF is stimulated by both hypoxia and O2-independent oncogenic, metabolic, and therapeutic factors. HIF drives angiogenesis by inducing secretion of proangiogenic growth factors by tumor cells and stromal cells, such as adipocytes and fibroblasts. The newly formed vasculature is disorganized and leaky, which facilitates tumor cell invasion and metastasis, impairs drug delivery, and further aggravates hypoxia in the tumor and the microenvironment. Angiogenic growth factors also contribute to an immunosuppressive tumor microenvironment, particularly by increasing recruitment of immunosuppressive cells. Compounds targeting angiogenic key players are listed in pink text. The key indicates their furthest stage of development in the breast cancer setting and evaluation in clinical trial(s) as monotherapy or as combination therapy. ANGPT(L), angiopoietin(-like) protein; BRCA, breast cancer gene; ER, estrogen receptor; FGFR, fibroblast growth factor receptor; HER, human epidermal growth factor receptor; MET, hepatocyte growth factor receptor; PARP, poly (ADP-ribose) polymerase; PTEN, phosphatase and tensin homolog; RET, rearranged during transfection; TAM, tumor-associated macrophage.

HIF-1 $\alpha$ immunohistochemistry in patient breast tumors correlates with ER $\alpha$ expression and HER2 positivity in some, but not all, studies $(11,18-22)$. High HIF-1 $\alpha$ levels are consistently reported in triple-negative breast cancer (TNBC), the poor-prognosis subtype that lacks (over)expression of hormonal and HER2 receptors (23-25). TNBC patients show especially high uptake of the PET tracer ${ }^{18} \mathrm{~F}$-fluoromisonidazole, which selectively accumulates in hypoxic cells (26), and TNBC cells carry a hypoxia gene signature in normoxic conditions (27). In TNBC, there is a high prevalence of p53 loss, PTEN mutations, and EGFR overexpression, all of which can lead to increased HIF activity (25). The transcription factor X-box binding protein 1 may regulate HIF responses in TNBC $(28,29)$. The lack of elevated HIFA mRNA levels in TNBC cells implies that important post-transcriptional mechanisms also contribute to the high HIF activity (27). Interestingly in this respect, intracellular depletion of the amino acid cysteine stabilizes HIF-1 $\alpha$ in TNBCs in normoxia and was associated with dysfunctional PHDs and paracrine glutamate signaling (23).

Multiple other metabolites and HIF-induced metabolic enzymes are involved in feed-forward loops with HIF activity in normoxia, including ROS, acetyl-CoA synthetase 2 (ACSS2), and mitochondrial proteins such as CHCHD4 (refs. 4, 30-33, and Figure 1). HIF $\alpha$ expression, stability, and effector function at HREs are additionally influenced by other (bidirectional) processes such as epigenetics, the circadian rhythm, noncoding RNAs, and HIF-dependent secretion of microvesicles by tumor cells or cells in the tumor microenvironment (TME) (9, 34-38). For instance, tumor-associated macrophages secrete vesicles containing the long noncoding RNA HISLA, which blocks the PHD/HIF-1 $\alpha$ interaction and induces glycolysis in 


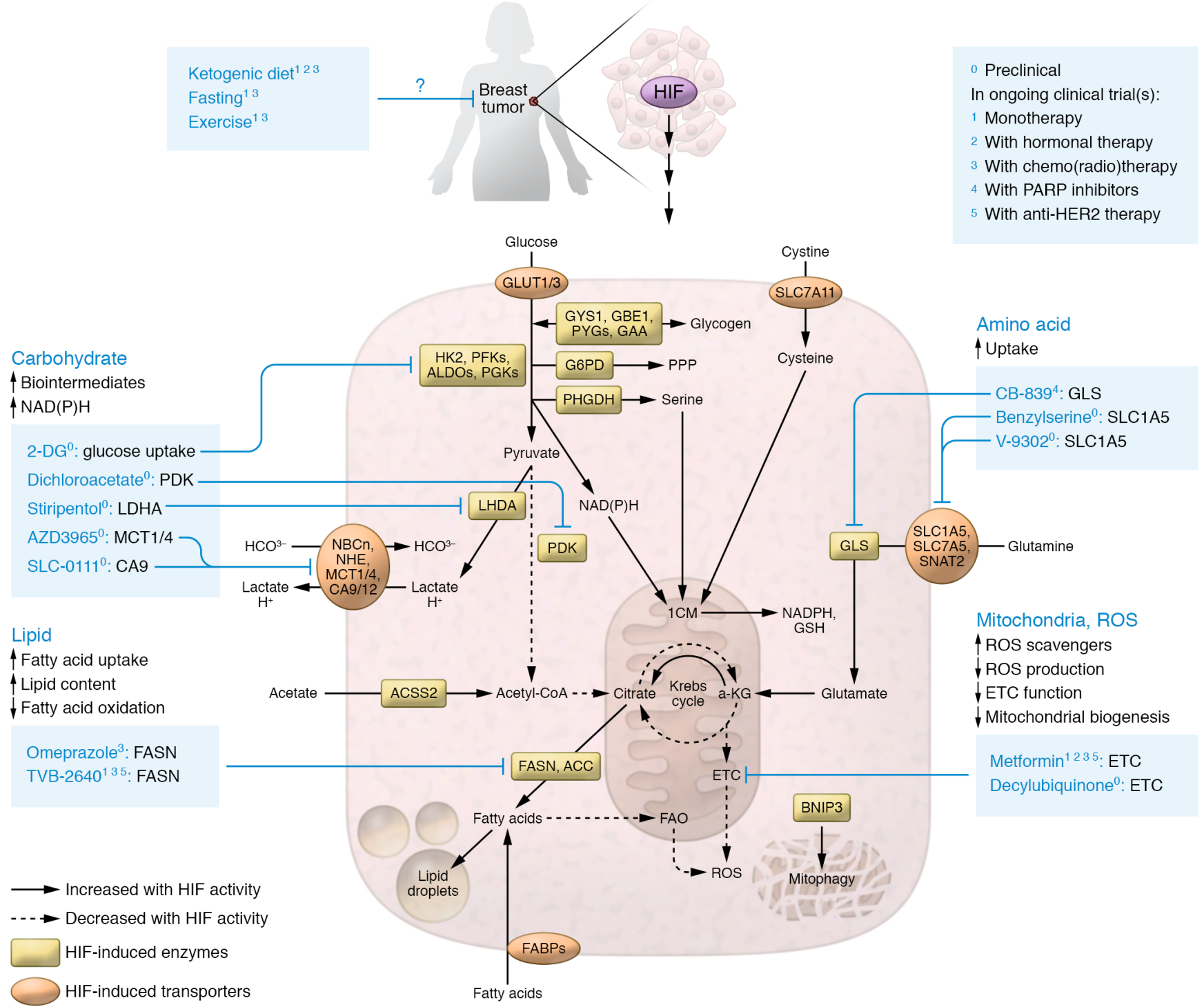

Figure 2. HIFs drive reprogramming of multiple metabolic pathways in breast cancer. In general, HIF activity increases glycolysis and related carbohydrate pathways (e.g. pentose phosphate pathway and glycogen metabolism) as well as lactate export while suppressing mitochondrial $\mathrm{O}_{2}$-dependent metabolism. Amino acid, acetate, and fatty acid uptake are increased to fuel processes that are essential for formation of ROS scavengers and Krebs cycle intermediates. This metabolic rewiring not only allows rapid proliferation and protects cells from ROS-induced damage but also contributes to formation of breast cancer stem cells and generation of an acidic and nutrient-depleted immunosuppressive microenvironment. Drugs with their respective targets or nonpharmaceutical, patient-centered strategies that target the rewired metabolism in breast cancer are listed in blue text. The key notes their furthest stage of (pre)clinical development in the breast cancer setting and/or evaluation in clinical trial(s) as monotherapy or as combination therapy. 1CM, one-carbon metabolism; 2-DC, 2-deoxyglucose; ACC, acetyl-CoA carboxylase; ACSS, acetyl-CoA synthetase; ALDO, aldolase; BNIP3, BCL2- and adenovirus E1B 19-kDa-interacting protein 3; CA, carbonic anhydrase; ETC, electron transport chain; FABP, fatty acid-binding protein; FAO, fatty acid oxidation; FASN, fatty acid synthase; G6PD, glucose-6-phosphate dehydrogenase; GAA, $\alpha$-1,4-glucosidase; GBE, glycogen branching enzyme; GLUT, glucose transporter; GSH, glutathione; GYS, glycogen synthase; HK, hexokinase; $\alpha$-KC, $\alpha$-ketoglutarate; LDHA, lactate dehydrogenase A; MCT, monocarboxylate transporter; NBC, Na+-bicarbonate cotransporter; NHE, $\mathrm{Na}^{+} / \mathrm{H}^{+}$exchanger; PDK, pyruvate dehydrogenase kinase; PFK, phosphofructokinase; PGK, phosphoglycerate kinase; PHCDH, phosphoglycerate dehydrogenase; PPP, pentose phosphate pathway; PYG, glycogen phosphorylase; SLC, solute carrier; SNAT, sodium-coupled neutral amino acid transporter.

normoxic breast cancer cells (35). HISLA secretion itself is increased by high extracellular lactate, demonstrating the intricate bidirectional pathways regulating $\mathrm{HIF} \alpha$ expression $(29,36,38)$.

\section{HIF-induced angiogenesis in breast cancer}

$\mathrm{O}_{2}$ diffusion from the nearest blood vessel, limited to a distance of 100 to $150 \mu \mathrm{m}$, typically supports tumor growth until it reach- es a volume of 1-2 $\mathrm{mm}^{3}$. Angiogenesis allows tumors to continue growing beyond sizes at which diffusion-mediated $\mathrm{O}_{2}$ and nutrient supplies fall short. HIF activity is the major driver of angiogenesis. The sprouting microvasculature in the TME is disorganized and leaky, in contrast to angiogenesis in normal tissue, and amplifies intratumoral hypoxia and favors metastatic spread while diminishing drug delivery and hampering antitumor immune responses 
Table 1. Selected studies reporting prognostic and/or predictive value of HIF and HIF targets in metabolism and angiogenesis in breast cancer patients

\begin{tabular}{|c|c|c|c|}
\hline Biomarker & Method & Prognostic for & Predictive for \\
\hline \multicolumn{4}{|l|}{ General HIF } \\
\hline HIF-1 $\alpha$ & $\mathrm{IHC}$ & $\begin{array}{l}\text { OS }(18,20) \\
\text { DFS }(18,20)\end{array}$ & $\begin{array}{l}\text { Neoadjuvant chemotherapy }(22,103,105) \\
\text { Antiestrogen }(175)\end{array}$ \\
\hline HIF-2 $\alpha$ & $\mathrm{IHC}$ & $\begin{array}{l}\text { DSS (176) } \\
\text { RFS (176) } \\
\text { OS (177) }\end{array}$ & - \\
\hline miR-210 & RNA sequencing & $\begin{array}{l}\text { OS (29) } \\
\text { Time to metastasis (29) }\end{array}$ & - \\
\hline Hypoxia gene signature & $\begin{array}{l}\text { RNA sequencing } \\
\text { Microarray }\end{array}$ & $\operatorname{OS}(27,99,100)$ & Antiangiogenic (80) \\
\hline (Peri)tumoral oxygen saturation & $\begin{array}{l}\text { Diffuse optical spectroscopy imaging } \\
{ }^{18} \mathrm{~F}-\mathrm{MISO} \mathrm{PET} / \mathrm{CT}\end{array}$ & - & $\begin{array}{l}\text { Neoadjuvant chemotherapy }\left(178^{A}, 179\right) \\
\text { Antiestrogen }(180)\end{array}$ \\
\hline \multicolumn{4}{|l|}{ Metabolism } \\
\hline $\begin{array}{l}\text { CA9 } \\
\text { pH regulation }\end{array}$ & $\begin{array}{l}\text { Serum measurement } \\
\text { IHC }\end{array}$ & $\begin{array}{l}\text { PFS }(181,182) \\
\text { DFS }(182,183) \\
\text { OS }(182) \\
\text { DSS }(183)\end{array}$ & (Neo)adjuvant chemotherapy $(183,184)$ \\
\hline $\begin{array}{l}\text { Glycolysis } \\
\text { Carbohydrate metabolism }\end{array}$ & $\begin{array}{l}\text { IHC (CLUT1, HK2 etc.) } \\
{ }^{18} \text { F-FDG PET/CT imaging }\end{array}$ & $\begin{array}{l}\text { DFS }(96,185) \\
\text { OS }(96)\end{array}$ & $\begin{array}{l}\text { (Neoadjuvant) anti-HER2 + chemotherapy }(115,116,186) \\
\text { Neoadjuvant chemotherapy }(103,187,188)\end{array}$ \\
\hline $\begin{array}{l}\text { NDRG1 } \\
\text { Fatty acid metabolism }\end{array}$ & $\begin{array}{l}\text { RNA sequencing } \\
\text { IHC }\end{array}$ & $\begin{array}{l}\text { RFS }(81,112) \\
\text { OS (112) }\end{array}$ & Antiangiogenic (80) \\
\hline $\begin{array}{l}\text { SLC7A5 } \\
\text { Amino acid metabolism }\end{array}$ & $\begin{array}{l}\text { RNA sequencing } \\
\text { IHC }\end{array}$ & $\begin{array}{l}\text { RFS }(111,112) \\
\text { OS }(111,112) \\
\text { DSS }(113)\end{array}$ & - \\
\hline $\begin{array}{l}\text { SLC1A5 } \\
\text { Amino acid metabolism }\end{array}$ & $\begin{array}{l}\text { IHC } \\
\text { RPPA }\end{array}$ & DFS (72) & - \\
\hline $\begin{array}{l}\text { SNAT2 } \\
\text { Amino acid metabolism }\end{array}$ & Gene array & - & Antiestrogen (66) \\
\hline $\begin{array}{l}\text { PHGDH } \\
\text { Amino acid/ROS metabolism }\end{array}$ & RNA sequencing & RFS (75) & - \\
\hline \multicolumn{4}{|l|}{ Angiogenesis } \\
\hline CXCR4 & $\mathrm{IHC} / \mathrm{IS} / \mathrm{WB}$ & $\begin{array}{l}\text { PFS (189) } \\
\text { OS }(189)^{A}\end{array}$ & - \\
\hline Microvessel density & $\mathrm{IHC}$ & $\begin{array}{l}\operatorname{RFS}\left(98^{A}, 190\right) \\
\text { OS }\left(98^{A}, 190\right)\end{array}$ & - \\
\hline VECFA & $\mathrm{IHC}$ & DFS (191) & - \\
\hline VEGFC & $\mathrm{IHC}$ & $\begin{array}{l}\text { OS }(191,192)^{A} \\
\text { DFS }(191,192)^{A}\end{array}$ & - \\
\hline VEGFR1 & $\mathrm{IHC}$ & DFS (191) & - \\
\hline MET & IHC/IS/RPPA/WB/FISH & $\begin{array}{l}\text { PFS }(189)^{A} \\
\text { OS }(124)^{A} \\
\text { RFS }(124)^{A}\end{array}$ & Adjuvant chemoradiotherapy (184) \\
\hline
\end{tabular}

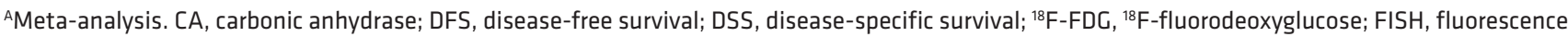
in situ hybridization; ${ }^{18} \mathrm{~F}$-MISO, ${ }^{18} \mathrm{~F}$-fluoromisonidazole; GLUT, glucose transporter; HK, hexokinase; IS, immunostaining; MET, hepatocyte growth factor receptor; NDRG, N-myc downstream regulated gene; OS, overall survival; PFS, progression-free survival; PHGDH, phosphoglycerate dehydrogenase; RFS, relapse-free survival; RPPA, reverse-phase protein array; SLC, solute carrier; SNAT, sodium-coupled neutral amino acid transporter; WB, Western blot.

(Figure 1 and refs. 39, 40). Breast cancer angiogenesis requires a well-balanced interplay between classical HIF-regulated angiogenic inducers (e.g., VEGF), angiogenic receptors (e.g., VEGFR, angiopoietin [ANGPT] receptors), and components of cell adhesion and extracellular matrix remodeling (41-43). Novel mediators of tumor angiogenesis are rapidly being identified (36). The long noncoding mRNA RAB11B-AS1 was increased in hypoxia in a HIF-2 $\alpha$-depen- dent manner and increased breast cancer angiogenesis and metastatic potential by recruiting RNA polymerase to VEGFA and angiopoietin-like 4 (ANGPTL4) (10). ANGPTL4 itself is a HIF-1 target that promotes lung metastasis when overexpressed in breast cancer cells (44). A recent breast cancer study in mice pointed toward adipocytes as an additional important source of ANGPTL4, and its secretion was synergistically controlled by hypoxia and IL-1 $\beta$ 


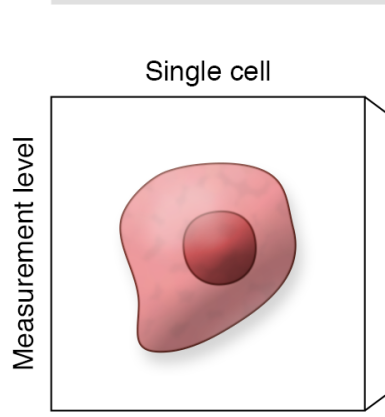

Single cell

Single-cell sequencing

Raman spectroscopy
Histology/immunohistochemistry

HIF- $1 / 2 \alpha$

Necrosis

Pimonidazole

Microvessel density

(e.g., $\mathrm{CD}^{31+}$, factor VIII)

VEGF(R)s, ANGPTLs, Tie2

Glycolytic markers

(e.g., CA9, GLUT1/3)
Cytology/experimental studies

Immunofluorescence

(e.g., hypoxia-sensitive probes)

Hypoxia-gene signature

(e.g., ChIP, RNA sequencing)

Angiogenesis assay

Seahorse

Metabolomics

(e.g., ${ }^{13} \mathrm{C}$ isotope tracing)

Extracellular $\mathrm{pH}$, lactate

Mitochondria

(e.g., membrane potential, count)

ROS production
Tumor with microenvironment

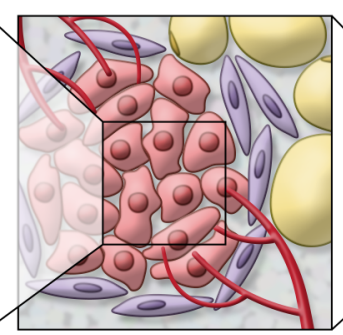

Imaging

$\mathrm{O}_{2}$-sensitive $\mathrm{PET} / \mathrm{CT}$ probes

(e.g., nitroimidazoles including ${ }^{18} \mathrm{~F}-\mathrm{MISO}$ and ${ }^{18} \mathrm{~F}-\mathrm{FAZA}, \mathrm{CU}-\mathrm{ATSM}$ )

Vasculature/bloodflow

(opticoacoustic sonography, ultrasound, DCE MRI)

Oxygen saturation

(BOLD MRI)

VEGF

(111/n/ ${ }^{19}$ Zr-bevacizumab PET/CT)

${ }^{18} \mathrm{FDG} \mathrm{PET} / \mathrm{CT}$

${ }^{1} \mathrm{H} M R S I$

Probe-based

$\mathrm{pO}_{2}$ histography

Blood-based

Serum levels (e.g., VEGF)
Hypoxia/general
Angiogenesis
Metabolism

Figure 3. Approaches to measure HIF activity, cancer angiogenesis, and metabolism. Depending on the method and the scale of application, various degrees of detail, intratumor and intrapatient heterogeneity, and interpatient heterogeneity can be captured. ANGPTL, angiopoietin-like protein; BOLD, blood oxygenation level-dependent; CA, carbonic anhydrase; Cu-ATSM, copper(II)-diacetyl-bis( $N^{4}$-methylthiosemicarbazone); DCE, dynamic contrastenhanced; ${ }^{18} \mathrm{~F}$-FAZA, ${ }^{18} \mathrm{~F}$-fluoroazomycin arabinoside; ${ }^{18} \mathrm{~F}$-FDG, ${ }^{18} \mathrm{~F}$-fluorodeoxyglucose; ${ }^{18} \mathrm{~F}-\mathrm{MISO},{ }^{18} \mathrm{~F}$-fluoromisonidazole; GEO, Gene Expression Omnibus; MRSI, magnetic resonance spectroscopic imaging; PET/CT, positron emission tomography/computed tomography; TCCA, The Cancer Genome Atlas; Tie2, TEK receptor tyrosine kinase 2.

$(45,46)$. Similarly, other studies reveal HIF-mediated release of (exosomal) proinflammatory and proangiogenic substances such as TGF- $\beta$ and prostaglandin $\mathrm{E}_{2}$ by breast cancer cells, adipocytes, infiltrating $\mathrm{CD}^{+} \mathrm{T}$ cells, and other stromal cells $(36,39,47-49)$, suggesting an intricate interplay between HIFs, proinflammatory factors derived from tumor and various TME cells, and angiogenesis that has yet to be fully elucidated.

\section{HIF-induced metabolic reprogramming in breast cancer}

Carbohydrate metabolism. HIF-1 activity induces a shift from respiratory, $\mathrm{O}_{2}$-dependent mitochondrial metabolism toward glycolytic, $\mathrm{O}_{2}$-independent metabolism through upregulation of nearly all glycolytic enzymes and redirection of pyruvate from entry into the Krebs cycle toward lactate production (refs. 4, 6, and Figure 2). Pyruvate dehydrogenase kinase (PDK) is a HIF-induced key regulator of lactate production via inhibition of pyruvate dehydrogenase ( $\mathrm{PDH}$ ), which rapidly inhibits the first step of the Krebs cycle during hypoxia (50).

These effects of HIF, which occur in hypoxia, are often confused with the Warburg effect, which is defined as aerobic glycolysis and is essential for formation of sufficient intermediates and reducing equivalents for rapid cell division and survival. Although normoxic HIF can mimic these effects, and HIF $\alpha$ may be upregulated by oncogenes, multiple other mechanisms are relevant, e.g., MYC and RAS (51). HIF not only induces glucose transporter (GLUT) expression for uptake of extracellular glucose 


\section{Table 2. Specific rationales for exploring synergy between approved breast cancer therapies and (novel) therapies targeting HIF/hypoxia, angiogenesis, and HIF-related metabolic reprogramming, as proposed or tested in the preclinical setting}

\begin{tabular}{ll}
$\begin{array}{l}\text { Approved therapy } \\
\text { Immune checkpoint } \\
\text { inhibition }\end{array}$ & $\begin{array}{l}\text { Mechanism of action } \\
\text { checkents inactivation of TILs by blocking immune }\end{array}$ \\
& \\
\hline $\begin{array}{l}\text { Radiotherapy } \\
\text { Chemotherapy }\end{array}$ & $\begin{array}{l}\text { Induces lethal DNA damage by ROS } \\
\text { Inducethal DNA damage }\end{array}$ \\
\hline Antiestrogen therapy & $\begin{array}{l}\text { Blocks constitutive growth signals from overexpressed } \\
\text { ER (ER antagonists) or endogenous estrogen production } \\
\text { (aromatase inhibitors) }\end{array}$ \\
\hline HER2-targeted therapy & $\begin{array}{l}\text { Blocks constitutive growth signals from overexpressed } \\
\text { HER2 and/or directs chemotherapy delivery }\end{array}$
\end{tabular}

Main rationale(s) for combination therapy

Exploit PD-L1 upregulation that is induced by HIF ${ }^{A}$

Enhance immune cell infiltration (TILs, dendritic cells) by normalizing vasculature ${ }^{B}$ Decrease (VEGF-mediated) induction of immunosuppressive subsets (e.g., Tregs, M2 macrophages) Exploit PD-1 and CTLA-4 upregulation that is induced by anti-VEGF treatment ${ }^{B}$ Decrease immunosuppressive TME by normalizing extracellular $\mathrm{pH}$ and suppressing tumor nutrient uptake

Enhance tumor oxygenation and ROS production by normalizing vasculature ${ }^{\mathrm{B}}$

Overcome/prevent (multidrug) resistance and BCSC induction ${ }^{\mathrm{A}}$ Increase chemotherapy delivery(?) $)^{\mathrm{B}}$

Concurrent hits in multiple cancer hallmarks

Overcome/prevent endocrine resistance by blocking compensatory HIF upregulation ${ }^{A}$

Decrease endocrine resistance by blocking amino acid metabolism ${ }^{\complement}$

Overcome/prevent T-DM1 resistance by reversing hypoxia-induced caveolin-1 relocation and drug internalization ${ }^{A}$
Refs

$119,147,149$,

150, 193-197

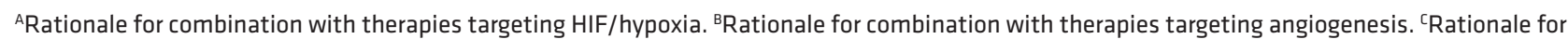
combination with therapies targeting HIF-related metabolic reprogramming. BCSC, breast cancer stem cell; CTLA-4, cytotoxic T Iymphocyte-associated protein 4; ER, estrogen receptor; HER2, human epidermal growth factor receptor 2; PD-1, programmed cell death protein 1; PD-L1, programmed death ligand 1; T-DM1, trastuzumab-emtansine; TIL, tumor-infiltrating lymphocyte; TME, tumor microenvironment.

but also increases glycogen synthesis and breakdown as an additional glucose source to sustain glycolytic and pentose phosphate flux. Breast cancer glycogen metabolism has been implicated in improved ROS scavenging, survival after reoxygenation, cell migration, and radioresistance (52).

HIF-induced membrane expression of lactate, $\mathrm{H}^{+}$, and $\mathrm{HCO}_{3}^{-}$ transporters is crucial for survival of hypoxic tumor cells by preventing intracellular $\mathrm{pH}$ reduction caused by lactate production, thereby allowing continuously high glycolytic rates and contributing to an acidic, immunosuppressive TME (53-55). While normal breast tissue does not express carbonic anhydrase 9 (CA9), it is widely overexpressed from DCIS (56) to invasive ductal carcinoma $(57,58)$ and lymph node metastases $(59,60)$. CA9 expression correlates well with tumor HIF-1 $\alpha$ activity and is particularly pronounced in perinecrotic tumor regions, high-grade breast cancers, and TNBC $(54,58,61)$. Besides the canonical CA function of catalyzing the interconversion of $\mathrm{CO}_{2}$ and water to $\mathrm{HCO}_{3}{ }^{-}$and $\mathrm{H}^{+}$ $(53,54)$, the noncatalytic domain of CA9 interacts with monocarboxylate transporters (MCTs) 1 and 4 in human breast cancer tissue, facilitating MCT-mediated lactate and $\mathrm{H}^{+}$efflux in preclinical models (62-65).

Amino acid metabolism. Amino acids, acetyl-CoA, and Krebs cycle intermediates are indispensable for nucleoside, lipid, and glutathione formation. To compensate for the reduced influx of pyruvate into the Krebs cycle, hypoxic cancer cells rely on uptake of amino acids such as glutamine and cystine to fuel this cycle. Glutamine, especially, has a central role in cancer cell metabolism. The amino acid importers SNAT2 (which imports neutral $\alpha$-amino acids including glutamine and alanine), solute-linked carrier family A1 member 5 (SLC1A5, also known as alanine, serine, cysteine transporter 2 [ASCT2], importing neutral amino acids, especially glutamine), SLC7A11 (a cystine-glutamate antiporter), and SLC7A5 (which mediates import of large neutral amino acids including leucine and tyrosine) and the enzyme glutaminase (GLS), which catalyzes glutamine-to-glutamate conversion, are all upregulated by HIF (refs. 66-70 and Figure 2). SLC1A5 was recently shown to be a HIF-2 target (68) and is especially overexpressed in TNBC. In vitro and in vivo SLC1A5 knockdown inhibits growth in TNBC, but not $\mathrm{ER} \alpha^{+}$breast cancer, sensitizes TNBC cells to chemotherapy, and is lethal in TNBCs that do not show a flexible compensatory increase in other amino acid transporters (71-73).

Serine, a nonessential amino acid derived from the glycolytic intermediate 3-phosphoglycerate, and cysteine are key for NADPH and glutathione formation in hypoxic breast cancer cells $(70,74,75)$. Phosphoglycerate dehydrogenase (PHGDH) and all other downstream enzymes in serine, cysteine, and downstream mitochondrial one-carbon metabolism are upregulated by HIF $(70,75)$. PHGDH knockdown in breast cancer cell lines reduces NADPH and glutathione levels, increases ROS levels, impairs metastatic potential by reducing breast cancer stem cells (BCSCs), and increases chemotherapy sensitivity. In contrast, breast cancer cell proliferation and growth are only impaired upon PHGDH knockdown in low-serine culture medium or in cell lines with a $P H G D H$ copy number gain (a small subset of TNBC). This implicates that breast cancer cells depend heavily on serine metabolism for ROS scavenging but are only dependent on it for biomass in case of intrinsic baseline PHGDH overexpression or serine-limiting environmental conditions $(75,76)$.

Lipid metabolism. Elevated levels of lipids and upregulation of fatty acid (FA) synthase (FASN) in breast cancer were the first observations consistent with the now well-established importance of lipid metabolism in cancer cells $(77,78)$. Cancer cells require FAs and lipids as building blocks for cell membranes, signaling 
molecules, energy, and reducing capacity during reoxygenation (77). HIF-1 activity represses FA oxidation, thereby reducing ROS generation, and upregulates FASN, lipin 1, acetyl-CoA carboxylase (ACC), and others for lipid and FA synthesis (Figure 2). Nevertheless, hypoxic cells are thought to preferably derive FAs from increased uptake by upregulating FA-binding proteins (FABPs), needed for FA uptake and intracellular trafficking, and predominantly use de novo lipid and FA synthesis from acetyl-CoA in nutrient-deprived conditions (77). Acetyl-CoA can be supplied through import of acetate, which is directly converted to acetyl-CoA in the cytoplasm by the HIF target ACSS2 $(6,71,77,79)$.

The HIF-regulated N-myc downstream regulated gene 1 (NDRG1) is predominantly overexpressed in perinecrotic areas and $\mathrm{ER} \alpha^{-}$breast cancer and is predictive for bevacizumab response and prognostic for survival $(80,81)$. Homozygous loss of function of NDRG1 in humans causes a neurological disorder with nerve demyelination, and manipulation of NDRG1 in breast cancer cell lines deregulated lipid droplet storage, although its exact metabolic function and discrepancies in its reported effects on migration and breast cancer progression require further clarification $(81,82)$.

Mitochondrial and ROS metabolism. ROS are produced due to dysfunction of the mitochondrial electron transport chain under hypoxic or hyperoxic conditions. In fact, in experimental hypoxia and HIF-KO models the prime cause of tumor cell death is ROS, rather than absolute $\mathrm{O}_{2}$ deficiency (83). HIFs keep intracellular ROS levels in check by increasing BCL2- and adenovirus E1B 19-kDa-interacting protein 3/Nip3-like protein X/FUN14 domain containing 1-mediated (BNIP3/NIX/FUNDC1-mediated) mitophagy, suppressing mitochondrial biogenesis, redirecting metabolic pathways to mitochondria-independent alternatives, and increasing production of the ROS scavenger glutathione and the reducing equivalent $\mathrm{NAD}(\mathrm{P}) \mathrm{H}$ (refs. 83-85 and Figure 2).

HIF-mediated suppression of nuclear respiratory factor 1 (NRF-1) decreases transcription of mitochondrial genes, and inhibition of the NRF-1 degrader SIAH2 (seven in absentia homolog 2; an E3 ubiquitin ligase) is associated with elevated $\mathrm{NAD}^{+} / \mathrm{NADH}$ ratios, succinate dehydrogenase activity, and increased mitochondrial mass $(85,86)$. Besides favoring breast cancer viability and growth, sublethal ROS levels stimulate HIF activity and induce cellular transformation into a BCSC phenotype, characterized by ongoing self-renewal capacity, stem cell markers such as aldehyde dehydrogenase (ALDH), and involvement in relapse and therapy resistance $(83,87)$. Moreover, HIF-1-dependent BCSC enrichment is observed upon chemotherapy treatment, and the majority of murine metastatic breast cancer cells exhibit a post-hypoxic, ROS-resistant phenotype even after reoxygenation (87-90).

\section{Biomarkers of HIF-regulated metabolism and angiogenesis}

A biomarker is defined as a characteristic that is objectively measured and evaluated as an indicator of normal biological processes, pathogenic processes, or pharmacological responses to a therapeutic intervention (91). Biomarkers can be prognostic, i.e., providing information on survival outcomes irrespective of the received treatment, and/or predictive, i.e., providing information on likelihood of treatment response. For instance, presence or absence of lymph node metastases is a strong prognostic but not a predictive marker, whereas the established breast cancer biomarkers HER2 overexpression and ER $\alpha$ expression are validated as prognostic as well as predictive biomarkers for response to HER2-targeted and hormonal therapy, respectively.

Multiple HIF-regulated angiogenic and metabolic tissue markers - either alone or in combination - have been implicated as prognostic for overall and progression-free survival and/or predictive for breast cancer chemotherapy, hormonal therapy, and kinase-targeted therapies (Table 1). Nevertheless, repeatability and clinical implementation of immunohistochemical markers are notoriously challenging, and study outcomes have been highly variable. Moreover, biopsy-based biomarkers are limited by sampling bias because they represent only a single part of a single tumor lesion. Imaging techniques can overcome this limitation by providing both static and dynamic whole-body measurements, albeit limited by their resolution. Noninvasive imaging approaches that measure real-time tumor blood flow or hemoglobin oxygen saturation or visualize trapped hypoxia-sensitive radioactive probes using PET could replace microvessel density (MVD) assessment, and whole-body ${ }^{18} \mathrm{~F}$-fluorodeoxyglucose $\left({ }^{18} \mathrm{~F}\right.$-FDG) PET/CT imaging may replace GLUT1 immunohistochemistry (refs. 92, 93, and Figure 3). The sections below discuss the most recent developments and previous studies that have been pioneering and/or included relatively large populations.

Prognostic markers. Tumor hypoxia has been measured mainly by determination of HIF- $1 \alpha$ expression and surrogates such as MVD and CA9 that are more stable than HIF-1 $\alpha$ itself, which has a half-life of $\leq 5$ minutes upon reoxygenation $(3,94)$. Presence of a hypoxic phenotype is prognostic for relapse and poor survival across breast cancer subtypes and stages, corroborated by well-powered pan-cancer meta-analyses $(95,96)$. The relative risks of high expression of HIF- $1 \alpha$, MVD, VEGF, CA9, and other hypoxic markers are only moderate compared with known clinical prognostic factors that already represent the aggressive phenotype associated with HIFs (e.g., receptor status, lymph node status, tumor grade). Contradictory results among studies are likely due to inconsistent multivariate correction, methodological differences in antibodies and targets for visualizing vascular endothelium (e.g., CD31 ${ }^{+}$, PDGF, factor VIII), variable scoring methodologies (e.g., manual vs. automated, nuclear vs. diffuse HIF $\alpha$ staining), and different stratification cutoffs $(97,98)$.

Rather than pinpointing of one marker, breast cancer HIF activity is increasingly captured by large-scale RNA sequencing in prognostic hypoxia-signature gene panels that contain components across multiple pathways downstream of $\operatorname{HIF}(27,99,100)$. This approach enhances the power to detect biologically relevant processes and guides discovery of new therapeutic targets and markers. Derived signatures can be validated in data sets publicly available online and in future studies. Genome-wide analysis of germline variations in almost 100,000 breast cancer patients in different cohorts revealed no major novel individual prognostic factors, whereas a network analysis identified the module "cell growth and angiogenesis" as prognostic for $\mathrm{ER} \alpha^{-}$but not $\mathrm{ER} \alpha^{+}$ breast cancer (101). One of the four components in this module was $C H C H D 4$, which encodes a mitochondrial protein involved in HIF- $1 \alpha$ stability and regulation of mitochondrial respiratory chain in tumor cell adaptation to hypoxia $(33,102)$. 
Predictive markers. It is generally acknowledged that tumor hypoxia and multiple HIF-related markers predict worse response to chemoradiotherapy, and neoadjuvant studies have shown lower pathological complete response (pCR) rates in patients with high baseline HIF $\alpha$ expression (22, 103-105). Several biological mechanisms explaining the negative correlation of HIF activity with chemoradiotherapy response have been described. Cytotoxicity of radiotherapy depends on ROS-induced catastrophic DNA damage, which therefore requires at least some $\mathrm{O}_{2}$. Additionally, the dysfunctional blood supply in hypoxic tumor regions may reduce delivery of cytotoxic drugs, and moreover, HIF upregulates P-glycoprotein, also called multidrug resistance protein $1(39,42,106)$. Finally, HIFs and chemotherapy both induce chemotherapy-resistant BCSCs (83, 87, 107). The gene panels Oncotype DX and MammaPrint are prognostic for survival and predictive for benefit from adjuvant chemotherapy in ER $\alpha^{+} \mathrm{HER} 2^{-}$breast cancer patients and are used in clinical decision making. Both panels consist of gene sets that include known HIF targets and/or players in tumor metabolism and angiogenesis such as matrix metalloproteinase 9 (MMP9) and egl-9 family hypoxia-inducible factor 1 (EGLN1), encoding PHD2 $(23,108)$. However, two of the control genes, GAPDH and TFRC (transferrin receptor), are well-validated HIF-1 targets, implying that differences driven by hypoxic tumor biology may be missed in these analyses $(109,110)$.

High expression of HIF- $1 \alpha$ and the HIF-regulated amino acid importers SNAT2, SLC1A5, and SLC7A5 has been associated with shorter survival in the $\mathrm{ER} \alpha^{+}$highly proliferative subtype (luminal B) and resistance to the antiestrogen therapies tamoxifen and aromatase inhibitors $(66,111-114)$. SNAT2 overexpression in hypoxic breast cancers is HIF- $1 \alpha$ - and HIF- $2 \alpha$-dependent and strongly corresponds with HIF1A mRNA expression and wider hypoxia gene signatures. SNAT2 has overlapping binding sites for HIF-1 $\alpha$ and $\mathrm{ER} \alpha$, and during tamoxifen treatment, which abolished ER $\alpha$ signaling, HIF-1 $\alpha$ could replace this signaling and increase SNAT2 expression under hypoxic conditions. SNAT2 knockdown reversed tamoxifen resistance and dampened signaling through the mTOR pathway, the latter being a known resistance mechanism to antiestrogen therapy (66). Other reports also describe a HIF-2 $\alpha$ and/or SLC7A5/mTOR regulatory axis underlying endocrine resistance $(9,67)$. In addition, contralateral breast cancers developing during adjuvant tamoxifen treatment, i.e., indicating intrinsic antiestrogen resistance, were more often HIF- $1 \alpha$-expressing than treatment-naive contralateral tumors (21).

The backbone of systemic therapy in breast cancer patients overexpressing HER2 are drugs that suppress the downstream oncogenic PI3K/Akt/mTOR and MAPK signaling pathways through HER2 inhibition and, in the case of the antibody-drug conjugate trastuzumab-emtansine (T-DM1), additionally deliver localized chemotherapy. The intensity of HER2 expression as determined by immunohistochemistry or FISH in tumor biopsies is the strongest predictive factor for therapy response, but intrinsic or induced resistance is a major clinical challenge that is not predicted by expression alone. ${ }^{18} \mathrm{~F}-\mathrm{FDG}$ uptake on $\mathrm{PET} / \mathrm{CT}$ is prognostic in the neoadjuvant and the metastatic setting for, respectively, pCR and early treatment failure (after approximately 2 cycles) $(115,116)$. Other markers of HIF-1/2 $\alpha$ expression or downstream metabolic or angiogenic targets have not been reported as predictive for response or resistance to HER2-targeted therapy.

The initial progression-free survival (PFS) gain in breast cancer demonstrated for the VEGF-targeting antibody bevacizumab did not translate into an overall survival (OS) benefit. It was subsequently reasoned that only patients with especially deregulated and widespread tumor microvasculature might benefit from bevacizumab-induced vessel normalization. However, in retrospective analyses, intuitively logical biomarkers correlated with $\mathrm{pCR}$ rates and normalization of tumor vasculature in some cases but did not predict final clinical outcomes. Evaluated biomarkers include high baseline MVD, high volume transfer constant on dynamic contrast-enhanced MRI, elevated expression of proangiogenic factors (e.g., VEGF, VEGFR, and Tie2 measured immunohistochemically or in patients' serum), and, more recently, NDRG1 and panels representing DNA methylation status or hypoxia gene sets in HER2- breast cancer patients on neoadjuvant bevacizumab plus chemotherapy $(5,80,117-119)$. Multiple alternative vascular markers are being evaluated in different cancer types, e.g., the vascular co-option players stromal-derived factor $1 \alpha$ and CXCR4, and ANGPT2 $(5,39)$.

\section{Targeting hypoxia, angiogenesis, and metabolism in breast cancer}

In breast cancer, hypoxia mediates aggressive, metastatic, and therapy-resistant disease, making it an attractive target for novel (combination) therapies (Table 2). Hypoxic tumor cells can be targeted directly, for example by use of hypoxia-activated prodrugs or by specific targeting of HIFs (reviewed in ref. 120). Strategies to target HIFs include downregulating HIF $\alpha$ protein expression, blocking HIF $\alpha$-HIF $\beta$ dimerization or essential cofactor binding, and preventing binding of HIF to HREs. It has, however, been challenging to develop specific, potent HIF-1 $\alpha$ inhibitors with suitable pharmacological properties for clinical evaluation. Review of ClinicalTrials.gov does not show any currently active breast cancer trials testing drugs directly targeting HIFs, although there are ongoing studies on (novel) inhibitors of mTOR (e.g., TAK-228), PI3K (e.g., BKM-120 or BYL-719), and histone deacetylases (vorinostat), which all indirectly target HIF signaling. Instead, therapeutic strategies often focus on consequences of hypoxia, including angiogenesis and reprogrammed metabolism, as discussed below (see also Figure 1 and Figure 2).

Therapeutic strategies targeting angiogenesis. The largest body of evidence is available for bevacizumab, a monoclonal antibody that blocks VEGF. As mentioned, in metastatic breast cancer only modest benefits in PFS were achieved, not translating into OS benefit, resulting in FDA withdrawal after initial approval. Targeting VEGF signal transduction with tyrosine kinase inhibitors is another strategy, but results in metastatic breast cancer are also disappointing (121). Although suppressing the VEGF pathway indeed decreases vascular density, rapid revascularization occurs within 2 weeks as shown in a neoadjuvant window-of-opportunity bevacizumab study $(5,39,119)$. This is likely mediated through induction of hypoxia by the antiangiogenic therapy, resulting in compensatory upregulation of both VEGF and VEGF-independent angiogenesis pathways $(119,122)$. Proposed resistance mechanisms include vascular mimicry, enhancement of invasive potential, recruitment of 
bone marrow-derived precursor endothelial cells, and promotion of alternative proangiogenic pathways $(5,39,42,123)$, which are of interest as potential therapeutic targets in breast cancer.

Hypoxia created by VEGF pathway inhibitors correlates with upregulation of the $M E T$ oncogene, which promotes invasive behavior and is an adverse prognostic factor in breast cancer (42, 123, 124). Cabozantinib (XL-184) is a potent oral inhibitor of MET and VEGFR2, and phase II trials showed mixed clinical benefit rates (0\%-34\%) in metastatic TNBC $(125,126)$.

In TNBC xenografts, dual FGF/VEGF targeting with or without paclitaxel chemotherapy showed synergistic effects in reducing vessel number and growth $(127,128)$. In a phase II trial of the dual FGF/VEGF inhibitor brivanib in solid tumors, responses were seen in breast cancer patients; however, this cohort was terminated early (129). Nintedanib, an inhibitor of VEGFR, PDGFR, and FGF receptors (FGFRs) that is approved for non-small cell lung cancer, showed preclinical activity in combination with paclitaxel in breast cancer xenografts and is being tested in breast cancer patients $(130,131)$. Interestingly, FGFR signaling also appears to mediate resistance to CDK4/6 inhibitors in breast cancer (132).

Trebananib (AMG386) is an ANGPT antagonist peptide-Fc fusion protein that selectively binds ANGPT1 and ANGPT2 (133). However, a phase II clinical trial in metastatic breast cancer patients indicated no evidence of benefit when combining AMG386 and paclitaxel with bevacizumab (133).

Src kinase is required for VEGF-induced proliferation of vascular cells, for vascular permeability, and for tumor cell extravasation in preclinical models (134). In phase II breast cancer studies, circulating VEGFR increased during exposure to the Src inhibitor dasatinib, implying that combination of VEGF and Src inhibitors may also be of interest (134).

Inhibition of angiogenesis may result in selection of cells that can use existing vasculature, known as co-option, a growth pattern observed in breast cancer liver metastases (135). In patients with colorectal cancer liver metastases, co-option was associated with poor response to bevacizumab (136). Inhibitors of key players in co-option such as the actin-related protein $2 / 3$ complex (Arp2/3), also expressed in breast cancer liver metastases, enhanced the efficacy of angiogenesis inhibitors in preclinical models of liver metastases (136).

Pharmaceutical targeting of metabolism in breast cancer. In preclinical breast cancer models, agents that directly interfere with high glucose uptake (e.g., the glucose analog 2-deoxyglucose) or decrease glycolysis (e.g., the PDK inhibitor dichloroacetate) reduced proliferation, inhibited HIF-1 $\alpha$, and sensitized cells to chemotherapy and mitochondrial inhibitors (137-139). Although phase I clinical cancer trials have included some breast cancer patients, toxicity has been a problem and no clear efficacy signals have emerged (140).

Lactate dehydrogenase (LDH) is a key enzyme for the interconversion of pyruvate and lactate. Although its complex biochemistry and multiple isoenzymes have made it hard to "drug" (141), several molecules are of interest for further development in cancer, including the old anticonvulsant stiripentol, which inhibits LDHA in vivo (142). Other ways to target lactate metabolism include blocking its transmembrane transport by inhibiting MCT1 and MCT4 (143-145). Inhibition of MCT1 in breast cancer was effective preclinically; however, the main mechanism appeared to be reduced pyruvate export rather than altered lactate transport or reduced glycolytic flux (146). The major immunosuppressive effect of extracellular lactate $(147,148)$ makes combinations of inhibitors of lactate transport with immune checkpoint inhibition of interest, especially in TNBC, in which checkpoint inhibition has proven effectiveness when combined with chemotherapy. Indeed, MCT1 blockade with AZD3965 increases immune cell infiltration in tumors, and inhibiting CA9 enhances immune responses to PD-L1 inhibition $(149,150)$. AZD3965 and the CA9 inhibitor SLC0111 are currently in phase I cancer trials.

Dependence of breast cancer cells on glutamine is increased not only in hypoxia but also in estrogen-independent and antiestrogen treatment-resistant subtypes (151). Preclinically, pharmacological targeting of HIF-regulated amino acid importers, for instance by the SLC1A5 inhibitors benzylserine or V-9302, blocks breast cancer cell growth and is associated with decreased mTOR signaling and increased ROS levels and autophagy (69, 71, 152, 153). Inhibition of GLS by CB-839 also inhibits growth of TNBC cells but not $\mathrm{ER} \alpha^{+}$breast cancer cells, which rely on GLS2 instead (154). Combining CB-839 with the mTOR inhibitor everolimus, however, does inhibit growth of endocrine-resistant breast cancer xenografts $(151,155)$. This is of interest since mTOR inhibition is already being used clinically in combination with hormonal therapy in $\mathrm{ER} \alpha^{+}$patients to prevent endocrine resistance. $\mathrm{CB}-839$ is now being evaluated in early clinical (breast) cancer trials.

Regarding cancer cell lipid metabolism, blocking FA synthesis has received the most attention, and, in vitro, inhibiting FASN reduced proliferation and induced apoptosis (77). TVB-2640 is a specific FASN inhibitor that has now proceeded into a phase II breast cancer trial. Interestingly, proton pump inhibitors such as omeprazole also inhibit FASN (156). The proton pump inhibitor omeprazole improved survival in metastatic breast cancer patients receiving chemotherapy, making repurposing of this FDA-approved class of drugs of interest, and further clinical evaluation is ongoing (157).

Targeting of components in the glycolytic pathway and vascular normalization induced by antiangiogenic therapy increase dependence of cancer cells on mitochondrial metabolism. Metformin, an AMPK activator that is a cornerstone in the treatment of type 2 diabetes, inhibits mitochondrial complex 1. More recently, it has also been shown to inhibit growth differentiation factor 15 (GDF15), a HIF-1 target (158). In the preclinical setting, metformin increased internalization of caveolin-1/T-DM1 and sensitivity to T-DM1 treatment through suppression of the HIF-responsive Akt/ MAPK pathway (159). Metformin is one of the main metabolically targeted drugs currently under investigation in breast cancer with (combination) trials ongoing in the setting of prevention and maintenance (160). However, so far no benefit of metformin has been demonstrated in randomized trials, which may be related to compensatory increases in glucose uptake and transcription of many genes involved in mitochondrial metabolism that occur already within 1-2 weeks of treatment (161).

In a phase $0 / \mathrm{I}$ randomized trial in HER2-, treatment-naive primary breast cancer patients, single-dose bevacizumab treatment was followed by randomization to treatment with the mitochondrial inhibitor ME-344 or placebo. In paired pre- and 
post-treatment biopsies, reduced proliferation was demonstrated in ME-344-treated patients, especially in the subgroup that had vascular normalization measured using ${ }^{18} \mathrm{~F}-\mathrm{FDG}$ PET (162). This illustrates the type of trial design and smart drug combinations that will be essential for further therapeutic development.

Several agents that target ROS are being studied alone or in combination, including decylubiquinone, an FDA-approved coenzyme $\mathrm{Q}_{10}$ analog that inhibits angiogenesis in breast cancer cells through a ROS-dependent mechanism (163).

Nonpharmaceutical targeting of metabolism in breast cancer. Nonpharmaceutical interventions that take advantage of the metabolic differences between cancer cells and normal cells, many mediated by HIF-dependent pathways, are also of interest. Exercise is of increasing importance in breast cancer care and is associated with decreased tumor growth and improved patient mental well-being and survival. Reduction of ROS is one of the multiple hypothesized underlying mechanisms (164). Of specific dietary interventions that have been proposed to have anticancer effects, ketogenic diets and fasting have received the most attention $(165,166)$.

Ketogenic diets are based on the premise that cancer cells are more dependent on glucose and have defective mitochondrial metabolism compared with normal cells. These diets are composed of high fat, moderate protein, and low carbohydrate content, resulting in increased fat metabolism. FAs are oxidized in the liver to acetyl-CoA, and any excess is converted into ketone bodies, mainly $\beta$-hydroxybutyrate. Normal tissues, in contrast to cancers, have the ability to use ketones as a source of energy, thus making these diets more detrimental to cancer cells. Many cancer trials have been initiated to investigate the ketogenic diet and have shown feasibility and reduced central obesity and insulin levels but no clear anticancer efficacy $(167,168)$. It is now well recognized that mitochondria continue to be functional in cancers, reducing the likelihood of large effect sizes. Furthermore, effects may be compensated by utilization of extracellular $\beta$-hydroxybutyrate by breast cancers for acetyl-CoA production (169).

Fasting decreases glucose, insulin, and IGF-1 levels while increasing FA breakdown and production of ketones, similar to the ketogenic diet $(166,170)$. Reducing IGF-1 reduces Akt signaling, and lower glucose increases AMPK activity. In 13 breast cancer patients, short-term fasting appeared to reduce hematological toxicity of neoadjuvant chemotherapy, possibly through faster recovery of DNA damage in PBMCs (171). Nevertheless, ketogenic and fasting diets are extremely challenging to adhere to, especially for cancer patients in whom malnutrition is detrimental to quality of life, response to therapy, and survival. Thus, although many behavioral modifications have a promising metabolic rationale exploiting the Warburg effect and ROS, strong and mechanistic proof for direct anticancer efficacy from translational studies is warranted.

\section{Concluding remarks}

HIFs and downstream angiogenic and metabolic alterations play a major role in breast cancer aggressiveness, progression, and therapy resistance but have proven to be notoriously difficult targets in the clinic. Novel druggable targets in HIF upstream regulatory pathways and downstream angiogenic and metabolic pathways are increasingly being identified. Continuous technological developments in (noninvasive) measurement of tumor glucose uptake, hypoxia, and vasculature now enable real-time in vivo monitoring of treatment-induced alterations. Approaches to clinically study the fate of metabolites are important for stratification and for understanding responses and escape mechanisms, and novel metabolic tools such as ${ }^{18} \mathrm{~F}$-glutamine PET/CT and ${ }^{13} \mathrm{C}$-metabolite flux tracing have been developed for clinical use or are in development, e.g., ${ }^{18} \mathrm{~F}$-labeled MCT inhibitors (161, 172-174). Smart incorporation of these tools into trials at baseline and interim time points can aid in successful translation of proposed antiangiogenic and metabolically targeted therapies to the clinic. Since the narrow therapeutic window and rapid emergence of escape mechanisms have posed major hurdles to monotherapies targeting these pathways, combination of novel antiangiogenic and metabolic drugs with existing therapies and nonpharmaceutical interventions seems most promising.

\section{Acknowledgments}

ALH has research funding from the Breast Cancer Research Foundation, Cancer Research UK (grant number A18974), and the Kennington Cancer Fund. MJ has research funding from the Dutch Cancer Society (Young Investigator Grant 10913/2017-1).

Address correspondence to: Adrian L. Harris, Molecular Oncology Laboratories, Weatherall Institute of Molecular Medicine, University of Oxford, Headley Way, Oxford, OX3 9DS United Kingdom. Email: adrian.harris@oncology.ox.ac.uk.
1. DeSantis CE, et al. Breast cancer statistics, 2019. CA Cancer JClin. 2019;69(6):438-451.

2. Vaupel P, Schlenger K, Knoop C, Höckel M. Oxygenation of human tumors: evaluation of tissue oxygen distribution in breast cancers by computerized $\mathrm{O} 2$ tension measurements. Cancer Res. 1991;51(12):3316-3322.

3. Wang GL, Jiang BH, Rue EA, Semenza GL. Hypoxia-inducible factor 1 is a basic-helixloop-helix-PAS heterodimer regulated by cellular O2 tension. Proc Natl Acad Sci U S A. 1995;92(12):5510-5514.

4. Semenza GL. HIF-1 mediates metabolic responses to intratumoral hypoxia and oncogenic mutations. J Clin Invest. 2013;123(9):3664-3671.

5. Harris AL. Clinical strategies to inhibit tumor vas- cularization. In: Ribatti D, Pezzella F, eds. Tumor Vascularization. Elsevier Science; 2020:147-175.

6. Samanta D, Semenza GL. Metabolic adaptation of cancer and immune cells mediated by hypoxia-inducible factors. Biochim Biophys Acta Rev Cancer. 2018;1870(1):15-22.

7. Schödel J, Mole DR, Ratcliffe PJ. Pan-genomic binding of hypoxia-inducible transcription factors. Biol Chem. 2013;394(4):507-517.

8. Smythies JA, et al. Inherent DNA-binding specificities of the HIF- $1 \alpha$ and HIF- $2 \alpha$ transcription factors in chromatin. EMBO Rep. 2019;20(1):e46401.

9. Fu X, et al. FOXA1 upregulation promotes enhancer and transcriptional reprogramming in endocrine-resistant breast cancer. Proc Natl Acad
Sci U S A. 2019;116(52):26823-26834.

10. Niu Y, et al. HIF2-induced long noncoding RNA RAB11B-AS1 promotes hypoxia-mediated angiogenesis and breast cancer metastasis. Cancer Res. 2020;80(5):964-975.

11. Bos R, et al. Levels of hypoxia-inducible factor-1 alpha during breast carcinogenesis. J Natl Cancer Inst. 2001;93(4):309-314.

12. Li AG, et al. BRCA1-IRIS promotes human tumor progression through PTEN blockade and HIF-1 $\alpha$ activation. Proc Natl Acad Sci U S A. 2018;115(41):E9600-E9609.

13. van der Groep P, et al. HIF-1 $\alpha$ overexpression in ductal carcinoma in situ of the breast in BRCA1 and BRCA2 mutation carriers. PLoS One. 2013;8(2):e56055. 
14. Laughner E, Taghavi P, Chiles K, Mahon PC, Semenza GL. HER2 (neu) signaling increases the rate of hypoxia-inducible factor 1alpha (HIF-1alpha) synthesis: novel mechanism for HIF-1-mediated vascular endothelial growth factor expression. Mol Cell Biol. 2001;21(12):3995-4004.

15. Jarman EJ, et al. HER2 regulates HIF- $2 \alpha$ and drives an increased hypoxic response in breast cancer. Breast Cancer Res. 2019;21(1):10.

16. Yang J, et al. Estrogen receptor- $\alpha$ directly regulates the hypoxia-inducible factor 1 pathway associated with antiestrogen response in breast cancer. Proc Natl Acad Sci U S A. 2015;112(49):15172-15177.

17. Fuady JH, Gutsche K, Santambrogio S, Varga Z, Hoogewijs D, Wenger RH. Estrogen-dependent downregulation of hypoxia-inducible factor (HIF)-2 $\alpha$ in invasive breast cancer cells. Oncotarget. 2016;7(21):31153-31165.

18. Schindl M, et al. Overexpression of hypoxia-inducible factor 1alpha is associated with an unfavorable prognosis in lymph node-positive breast cancer. Clin Cancer Res. 2002;8(6):1831-1837.

19. Giatromanolaki A, et al. c-erbB-2 related aggressiveness in breast cancer is hypoxia inducible factor-1alpha dependent. Clin Cancer Res. 2004;10(23):7972-7977.

20. Bos R, et al. Levels of hypoxia-inducible factor-1alpha independently predict prognosis in patients with lymph node negative breast carcinoma. Cancer. 2003;97(6):1573-1581.

21. Jögi A, Ehinger A, Hartman L, Alkner S. Expression of HIF-1 $\alpha$ is related to a poor prognosis and tamoxifen resistance in contralateral breast cancer. PLoS One. 2019;14(12):e0226150.

22. Nie C, Lv H, Bie L, Hou H, Chen X. Hypoxia-inducible factor 1-alpha expression correlates with response to neoadjuvant chemotherapy in women with breast cancer. Medicine (Baltimore). 2018;97(51):e13551.

23. Briggs KJ, et al. Paracrine induction of HIF by glutamate in breast cancer: EglN1 senses cysteine. Cell. 2016;166(1):126-139.

24. Bane AL, et al. Tumor factors predictive of response to hypofractionated radiotherapy in a randomized trial following breast conserving therapy. Ann Oncol. 2014;25(5):992-998.

25. Cancer Genome Atlas Network. Comprehensive molecular portraits of human breast tumours. Nature. 2012;490(7418):61-70.

26. Asano A, et al. Intracellular hypoxia measured by ${ }^{18} \mathrm{~F}$-fluoromisonidazole positron emission tomography has prognostic impact in patients with estrogen receptor-positive breast cancer. Breast Cancer Res. 2018;20(1):78.

27. Ye IC, Fertig EJ, DiGiacomo JW, Considine M, Godet I, Gilkes DM. Molecular portrait of hypoxia in breast cancer: a prognostic signature and novel HIF-regulated genes. Mol Cancer Res. 2018;16(12):1889-1901.

28. Chen X, et al. XBP1 promotes triple-negative breast cancer by controlling the HIF1 $\alpha$ pathway. Nature. 2014;508(7494):103-107.

29. Liang H, et al. Hypoxia induces miR-153 through the IRE1 $\alpha$-XBP1 pathway to fine tune the HIF1 $\alpha$ / VEGFA axis in breast cancer angiogenesis. Oncogene. 2018;37(15):1961-1975.

30. Kappler M, et al. Causes and consequences of a glutamine induced normoxic HIF1 activity for the tumor metabolism. Int JMol Sci. 2019;20(19):E4742.

31. Grandjean G, et al. Definition of a novel feed-forward mechanism for glycolysis-HIF1 $\alpha$ signaling in hypoxic tumors highlights aldolase $\mathrm{A}$ as a therapeutic target. Cancer Res. 2016;76(14):4259-4269.

32. Xiong G, et al. Collagen prolyl 4-hydroxylase 1 is essential for HIF- $1 \alpha$ stabilization and TNBC chemoresistance. Nat Commun. 2018;9(1):4456.

33. Yang J, et al. Human CHCHD4 mitochondrial proteins regulate cellular oxygen consumption rate and metabolism and provide a critical role in hypoxia signaling and tumor progression. JClin Invest. 2012;122(2):600-611.

34. Wang T, et al. Hypoxia-inducible factors and RAB22A mediate formation of microvesicles that stimulate breast cancer invasion and metastasis. Proc Natl Acad SciU S A. 2014;111(31):E3234-E3242.

35. Chen F, et al. Extracellular vesicle-packaged HIF-1 $\alpha$-stabilizing lncRNA from tumour-associated macrophages regulates aerobic glycolysis of breast cancer cells. Nat Cell Biol. 2019;21(4):498-510.

36. Choudhry H, Harris AL. Advances in hypoxia-inducible factor biology. Cell Metab. 2018;27(2):281-298.

37. Sulli G, Lam MTY, Panda S. Interplay between circadian clock and cancer: new frontiers for cancer treatment. Trends Cancer. 2019;5(8):475-494.

38. Chen Y, et al. ZMYND8 acetylation mediates HIF-dependent breast cancer progression and metastasis. JClin Invest. 2018;128(5):1937-1955.

39. Martin JD, Seano G, Jain RK. Normalizing function of tumor vessels: progress, opportunities, and challenges. Annu Rev Physiol. 2019;81:505-534.

40. Khan KA, Kerbel RS. Improving immunotherapy outcomes with anti-angiogenic treatments and vice versa. Nat Rev Clin Oncol. 2018;15(5):310-324.

41. Wang JC, et al. Metformin inhibits metastatic breast cancer progression and improves chemosensitivity by inducing vessel normalization via PDGF-B downregulation. J Exp Clin Cancer Res. 2019;38(1):235.

42. Jayson GC, Kerbel R, Ellis LM, Harris AL. Antiangiogenic therapy in oncology: current status and future directions. Lancet. 2016;388(10043):518-529.

43. Carmeliet P, Jain RK. Molecular mechanisms and clinical applications of angiogenesis. Nature. 2011;473(7347):298-307.

44. Zhang H, et al. HIF-1-dependent expression of angiopoietin-like 4 and L1CAM mediates vascular metastasis of hypoxic breast cancer cells to the lungs. Oncogene. 2012;31(14):1757-1770.

45. Rausch LK, Netzer NC, Hoegel J, Pramsohler S. The linkage between breast cancer, hypoxia, and adipose tissue. Front Oncol. 2017;7:211.

46. Kolb R, et al. Obesity-associated inflammation promotes angiogenesis and breast cancer via angiopoietin-like 4. Oncogene. 2019;38(13):2351-2363.

47. Semenza GL. The hypoxic tumor microenvironment: A driving force for breast cancer progression. Biochim Biophys Acta. 2016;1863(3):382-391.

48. Incio J, et al. Obesity promotes resistance to antiVEGF therapy in breast cancer by up-regulating IL-6 and potentially FGF-2. Sci Transl Med. 2018;10(432):eaag0945.

49. Palazon A, et al. An HIF-1 $\alpha /$ VEGF-A axis in cytotoxic $\mathrm{T}$ cells regulates tumor progression. Cancer
Cell. 2017;32(5):669-683.e5.

50. Kim JW, Tchernyshyov I, Semenza GL, Dang CV. HIF-1-mediated expression of pyruvate dehydrogenase kinase: a metabolic switch required for cellular adaptation to hypoxia. Cell Metab. 2006;3(3):177-185.

51. Koppenol WH, Bounds PL, Dang CV. Otto Warburg's contributions to current concepts of cancer metabolism. Nat Rev Cancer. 2011;11(5):325-337.

52. Altemus MA, et al. Breast cancers utilize hypoxic glycogen stores via PYGB, the brain isoform of glycogen phosphorylase, to promote metastatic phenotypes. PLoS One. 2019;14(9):e0220973.

53. Pastorek J, et al. Cloning and characterization of $\mathrm{MN}$, a human tumor-associated protein with a domain homologous to carbonic anhydrase and a putative helix-loop-helix DNA binding segment. Oncogene. 1994;9(10):2877-2888 .

54. Wykoff CC, et al. Hypoxia-inducible expression of tumor-associated carbonic anhydrases. Cancer Res. 2000;60(24):7075-7083.

55. Boedtkjer E. $\mathrm{Na}^{+}, \mathrm{HCO}_{3}{ }_{3}^{-}$cotransporter $\mathrm{NBCn} 1$ accelerates breast carcinogenesis. Cancer Metastasis Rev. 2019;38(1-2):165-178.

56. Wykoff CC, et al. Expression of the hypoxia-inducible and tumor-associated carbonic anhydrases in ductal carcinoma in situ of the breast. Am J Pathol. 2001;158(3):1011-1019.

57. Chia SK, et al. Prognostic significance of a novel hypoxia-regulated marker, carbonic anhydrase IX, in invasive breast carcinoma. J Clin Oncol. 2001;19(16):3660-3668.

58. Adams A, et al. The potential of hypoxia markers as target for breast molecular imaging--a systematic review and meta-analysis of human marker expression. BMC Cancer. 2013;13:538.

59. Van den Eynden GG, et al. Angiogenesis and hypoxia in lymph node metastases is predicted by the angiogenesis and hypoxia in the primary tumour in patients with breast cancer. $\mathrm{Br} \mathrm{JCan}$ cer. 2005;93(10):1128-1136.

60. Tafreshi NK, et al. Noninvasive detection of breast cancer lymph node metastasis using carbonic anhydrases IX and XII targeted imaging probes. Clin Cancer Res. 2012;18(1):207-219.

61. Choi J, Jung WH, Koo JS. Metabolism-related proteins are differentially expressed according to the molecular subtype of invasive breast cancer defined by surrogate immunohistochemistry. Pathobiology. 2013;80(1):41-52.

62. Ames S, Andring JT, McKenna R, Becker HM. CAIX forms a transport metabolon with monocarboxylate transporters in human breast cancer cells. Oncogene. 2020;39(8):1710-1723.

63. Ames S, Pastorekova S, Becker HM. The proteoglycan-like domain of carbonic anhydrase IX mediates non-catalytic facilitation of lactate transport in cancer cells. Oncotarget. 2018;9(46):27940-27957.

64. Jamali S, et al. Hypoxia-induced carbonic anhydrase IX facilitates lactate flux in human breast cancer cells by non-catalytic function. Sci Rep. 2015;5:13605.

65. Mboge MY, et al. A non-catalytic function of carbonic anhydrase IX contributes to the glycolytic phenotype and $\mathrm{pH}$ regulation in human breast cancer cells. Biochem J. 2019;476(10):1497-1513. 66. Morotti M, et al. Hypoxia-induced switch in 
SNAT2/SLC38A2 regulation generates endocrine resistance in breast cancer. Proc Natl Acad Sci U S A. 2019;116(25):12452-12461.

67. Elorza A, et al. HIF2 $\alpha$ acts as an mTORC1 activator through the amino acid carrier SLC7A5. Mol Cell. 2012;48(5):681-691.

68. Yoo HC, et al. A variant of SLC1A5 is a mitochondrial glutamine transporter for metabolic reprogramming in cancer cells. Cell Metab. 2020;31(2):267-283.e12.

69. Altman BJ, Stine ZE, Dang CV. From Krebs to clinic: glutamine metabolism to cancer therapy. Nat Rev Cancer. 2016;16(10):619-634.

70. Lu H, et al. Chemotherapy triggers HIF-1-dependent glutathione synthesis and copper chelation that induces the breast cancer stem cell phenotype. Proc Natl Acad Sci U S A. 2015;112(33):E4600-E4609.

71. van Geldermalsen M, et al. ASCT2/SLC1A5 controls glutamine uptake and tumour growth in triple-negative basal-like breast cancer. Oncogene. 2016;35(24):3201-3208.

72. Jeon YJ, et al. Regulation of glutamine carrier proteins by RNF 5 determines breast cancer response to ER stress-inducing chemotherapies. Cancer Cell. 2015;27(3):354-369.

73. Bröer A, et al. Ablation of the ASCT2 (SLC1A5) gene encoding a neutral amino acid transporter reveals transporter plasticity and redundancy in cancer cells. JBiol Chem. 2019;294(11):4012-4026.

74. Ye J, et al. Serine catabolism regulates mitochondrial redox control during hypoxia. Cancer Discov. 2014;4(12):1406-1417.

75. Samanta D, Park Y, Andrabi SA, Shelton LM, Gilkes DM, Semenza GL. PHGDH expression is required for mitochondrial redox homeostasis, breast cancer stem cell maintenance, and lung metastasis. Cancer Res. 2016;76(15):4430-4442.

76. Sullivan MR, et al. Increased serine synthesis provides an advantage for tumors arising in tissues where serine levels are limiting. Cell Metab. 2019;29(6):1410-1421.e4.

77. Röhrig F, Schulze A. The multifaceted roles of fatty acid synthesis in cancer. Nat Rev Cancer. 2016;16(11):732-749.

78. Szutowicz A, Kwiatkowski J, Angielski S. Lipogenetic and glycolytic enzyme activities in carcinoma and nonmalignant diseases of the human breast. Br J Cancer. 1979;39(6):681-687.

79. Bharti SK, et al. Metabolic consequences of HIF silencing in a triple negative human breast cancer xenograft. Oncotarget. 2018;9(20):15326-15339.

80. Karn T, et al. A small hypoxia signature predicted $\mathrm{pCR}$ response to bevacizumab in the neoadjuvant GeparQuinto breast cancer trial. Clin Cancer Res. 2020;26(8):1896-1904.

81. Sevinsky CJ, Khan F, Kokabee L, Darehshouri A, Maddipati KR, Conklin DS. NDRG1 regulates neutral lipid metabolism in breast cancer cells. Breast Cancer Res. 2018;20(1):55.

82. Godbole M, et al. Up-regulation of the kinase gene $S G K 1$ by progesterone activates the AP-1-NDRG1 axis in both PR-positive and -negative breast cancer cells. J Biol Chem. 2018;293(50):19263-19276.

83. Xiang L, Semenza GL. Hypoxia-inducible factors promote breast cancer stem cell specification and maintenance in response to hypoxia or cytotoxic chemotherapy. Adv Cancer Res. 2019;141:175-212.

84. Tang K, et al. Hypoxia-reprogrammed tricarboxylic acid cycle promotes the growth of human breast tumorigenic cells. Oncogene. 2019;38(44):6970-6984.

85. Ma B, et al. The SIAH2-NRF1 axis spatially regulates tumor microenvironment remodeling for tumor progression. Nat Commun. 2019;10(1):1034.

86. Zhang J, et al. EglN2 associates with the NRF1-PGC1 $\alpha$ complex and controls mitochondrial function in breast cancer. $E M B O J$. 2015;34(23):2953-2970.

87. Semenza GL. Hypoxia-inducible factors: coupling glucose metabolism and redox regulation with induction of the breast cancer stem cell phenotype. ЕМВО J. 2017;36(3):252-259.

88. Godet I, Shin YJ, Ju JA, Ye IC, Wang G, Gilkes DM. Fate-mapping post-hypoxic tumor cells reveals a ROS-resistant phenotype that promotes metastasis. Nat Commun. 2019;10(1):4862.

89. Lee KM, et al. MYC and MCL1 cooperatively promote chemotherapy-resistant breast cancer stem cells via regulation of mitochondrial oxidative phosphorylation. Cell Metab. 2017;26(4):633-647.e7.

90. Msaki A, et al. A hypoxic signature marks tumors formed by disseminated tumor cells in the BALBneuT mammary cancer model. Oncotarget. 2016;7(22):33081-33095.

91. Biomarkers Definitions Working Group. Biomarkers and surrogate endpoints: preferred definitions and conceptual framework. Clin Pharmacol Ther. 2001;69(3):89-95.

92. Daimiel I. Insights into hypoxia: non-invasive assessment through imaging modalities and its application in breast cancer. J Breast Cancer. 2019;22(2):155-171.

93. Walsh JC, Lebedev A, Aten E, Madsen K, Marciano L, Kolb HC. The clinical importance of assessing tumor hypoxia: relationship of tumor hypoxia to prognosis and therapeutic opportunities. Antioxid Redox Signal. 2014;21(10):1516-1554.

94. Sobhanifar S, Aquino-Parsons C, Stanbridge EJ, Olive P. Reduced expression of hypoxia-inducible factor-1alpha in perinecrotic regions of solid tumors. Cancer Res. 2005;65(16):7259-7266.

95. Han S, Huang T, Hou F, Yao L, Wang X, Wu X. The prognostic value of hypoxia-inducible factor-1 $\alpha$ in advanced cancer survivors: a meta-analysis with trial sequential analysis. Ther Adv Med Oncol. 2019;11:1758835919875851.

96. Yu M, et al. Prognostic role of glycolysis for cancer outcome: evidence from 86 studies. J Cancer Res Clin Oncol. 2019;145(4):967-999.

97. Nowak-Sliwinska P, et al. Consensus guidelines for the use and interpretation of angiogenesis assays. Angiogenesis. 2018;21(3):425-532.

98. Uzzan B, Nicolas P, Cucherat M, Perret GY. Microvessel density as a prognostic factor in women with breast cancer: a systematic review of the literature and meta-analysis. Cancer Res. 2004;64(9):2941-2955.

99. Abu-Jamous B, Buffa FM, Harris AL, Nandi AK. In vitro downregulated hypoxia transcriptome is associated with poor prognosis in breast cancer. Mol Cancer. 2017;16(1):105.

100.Ye Y, et al. Characterization of hypoxia-associated molecular features to aid hypoxia-targeted thera- py. Nat Metab. 2019;1(4):431-444.

101.Escala-Garcia M, et al. A network analysis to identify mediators of germline-driven differences in breast cancer prognosis. Nat Commun. 2020;11(1):312.

102. Thomas LW, et al. CHCHD4 confers metabolic vulnerabilities to tumour cells through its control of the mitochondrial respiratory chain. Cancer Metab. 2019;7:2.

103. Milani M, et al. Hypoxia-related biological markers as predictors of epirubicin-based treatment responsiveness and resistance in locally advanced breast cancer. Oncotarget. 2017;8(45):78870-78881.

104.Generali D, et al. Hypoxia-inducible factor-1alpha expression predicts a poor response to primary chemoendocrine therapy and disease-free survival in primary human breast cancer. Clin Cancer Res. 2006;12(15):4562-4568.

105. Koukourakis MI, et al. Prospective neoadjuvant analysis of PET imaging and mechanisms of resistance to Trastuzumab shows role of HIF1 and autophagy. Br JCancer. 2014;110(9):2209-2216.

106.Samanta D, Gilkes DM, Chaturvedi P, Xiang L, Semenza GL. Hypoxia-inducible factors are required for chemotherapy resistance of breast cancer stem cells. Proc Natl Acad Sci U S A. 2014;111(50):E5429-E5438.

107. Brooks DL, et al. ITGA6 is directly regulated by hypoxia-inducible factors and enriches for cancer stem cell activity and invasion in metastatic breast cancer models. Mol Cancer. 2016;15:26.

108. Schito L, Rey S. Hypoxic pathobiology of breast cancer metastasis. Biochim Biophys Acta Rev Cancer. 2017;1868(1):239-245.

109. Rolfs A, Kvietikova I, Gassmann M, Wenger RH. Oxygen-regulated transferrin expression is mediated by hypoxia-inducible factor-1. J Biol Chem . 1997;272(32):20055-20062.

110. Higashimura $\mathrm{Y}$, et al. Up-regulation of glyceraldehyde-3-phosphate dehydrogenase gene expression by HIF-1 activity depending on Sp1 in hypoxic breast cancer cells. Arch Biochem Biophys. 2011;509(1):1-8.

111. Mihály Z, et al. A meta-analysis of gene expression-based biomarkers predicting outcome after tamoxifen treatment in breast cancer. Breast Cancer Res Treat. 2013;140(2):219-232.

112. Bartlett JM, et al. Mammostrat as a tool to stratify breast cancer patients at risk of recurrence during endocrine therapy. Breast Cancer Res. 2010;12(4):R47.

113. El Ansari R, et al. The amino acid transporter SLC7A5 confers a poor prognosis in the highly proliferative breast cancer subtypes and is a key therapeutic target in luminal B tumours. Breast Cancer Res. 2018;20(1):21.

114. Chen Z, Wang Y, Warden C, Chen S. Cross-talk between ER and HER2 regulates c-MYC-mediated glutamine metabolism in aromatase inhibitor resistant breast cancer cells. J Steroid Biochem Mol Biol. 2015;149:118-127.

115. Gebhart G, et al. ${ }^{18} \mathrm{~F}-\mathrm{FDG}$ PET/CT for early prediction of response to neoadjuvant lapatinib, trastuzumab, and their combination in HER2-positive breast cancer: results from Neo-ALTTO. J Nucl Med. 2013;54(11):1862-1868.

116. Gebhart G, et al. Molecular imaging as a tool 
to investigate heterogeneity of advanced HER2-positive breast cancer and to predict patient outcome under trastuzumab emtansine (T-DM1): the ZEPHIR trial. Ann Oncol. 2016;27(4):619-624.

117. Miles D, et al. Bevacizumab plus paclitaxel versus placebo plus paclitaxel as first-line therapy for HER2-negative metastatic breast cancer (MERiDiAN): a double-blind placebo-controlled randomised phase III trial with prospective biomarker evaluation. Eur JCancer. 2017;70:146-155.

118. Gampenrieder SP, et al. DNA methylation signatures predicting bevacizumab efficacy in metastatic breast cancer. Theranostics. 2018;8(8):2278-2288.

119. Mehta S, et al. Radiogenomics monitoring in breast cancer identifies metabolism and immune checkpoints as early actionable mechanisms of resistance to anti-angiogenic treatment. EBioMedicine. 2016;10:109-116.

120.Wigerup C, Påhlman S, Bexell D. Therapeutic targeting of hypoxia and hypoxia-inducible factors in cancer. Pharmacol Ther. 2016;164:152-169.

121. Barrios $\mathrm{CH}$, et al. Phase III randomized trial of sunitinib versus capecitabine in patients with previously treated HER2-negative advanced breast cancer. Breast Cancer Res Treat. 2010;121(1):121-131.

122.Ueda S, Saeki T, Osaki A, Yamane T, Kuji I. Bevacizumab induces acute hypoxia and cancer progression in patients with refractory breast cancer: multimodal functional imaging and multiplex cytokine analysis. Clin Cancer Res. 2017;23(19):5769-5778.

123. Mahdi A, Darvishi B, Majidzadeh-AK, Salehi M, Farahmand L. Challenges facing antiangiogenesis therapy: the significant role of hypoxia-inducible factor and MET in development of resistance to anti-vascular endothelial growth factor-targeted therapies. JCell Physiol. 2019;234(5):5655-5663.

124. Yan S, Jiao X, Zou H, Li K. Prognostic significance of c-Met in breast cancer: a meta-analysis of 6010 cases. Diagn Pathol. 2015;10:62.

125. Tolaney SM, et al. Phase II and biomarker study of cabozantinib in metastatic triple-negative breast cancer patients. Oncologist. 2017;22(1):25-32.

126. Leone JP, et al. A phase II study of cabozantinib alone or in combination with trastuzumab in breast cancer patients with brain metastases. Breast Cancer Res Treat. 2020;179(1):113-123.

127. Bello E, et al. The tyrosine kinase inhibitor E-3810 combined with paclitaxel inhibits the growth of advanced-stage triple-negative breast cancer xenografts. Mol Cancer Ther. 2013;12(2):131-140.

128. Golfmann K, et al. Synergistic anti-angiogenic treatment effects by dual FGFR1 and VEGFR1 inhibition in FGFR1-amplified breast cancer. Oncogene. 2018;37(42):5682-5693.

129. Jones RL, et al. Phase II randomised discontinuation trial of brivanib in patients with advanced solid tumours. Eur J Cancer. 2019;120:132-139.

130. Quintela-Fandino M, et al. Nintedanib plus letrozole in early breast cancer: a phase $0 / \mathrm{I}$ pharmacodynamic, pharmacokinetic, and safety clinical trial of combined FGFR1 and aromatase inhibition. Breast Cancer Res. 2019;21(1):69.

131. Reguera-Nuñez E, Xu P, Chow A, Man S, Hilberg F, Kerbel RS. Therapeutic impact of Nintedan- ib with paclitaxel and/or a PD-L1 antibody in preclinical models of orthotopic primary or metastatic triple negative breast cancer. J Exp Clin Cancer Res. 2019;38(1):16.

132. Formisano L, et al. Aberrant FGFR signaling mediates resistance to CDK4/6 inhibitors in ER+ breast cancer. Nat Commun. 2019;10(1):1373.

133. Diéras V, et al. Trebananib (AMG 386) plus weekly paclitaxel with or without bevacizumab as first-line therapy for HER2-negative locally recurrent or metastatic breast cancer: a phase 2 randomized study. Breast. 2015;24(3):182-190.

134. Mayer EL, Krop IE. Advances in targeting SRC in the treatment of breast cancer and other solid malignancies. Clin Cancer Res. 2010;16(14):3526-3532.

135. Stessels F, et al. Breast adenocarcinoma liver metastases, in contrast to colorectal cancer liver metastases, display a non-angiogenic growth pattern that preserves the stroma and lacks hypoxia. Br JCancer. 2004;90(7):1429-1436.

136. Frentzas $S$, et al. Vessel co-option mediates resistance to anti-angiogenic therapy in liver metastases. Nat Med. 2016;22(11):1294-1302.

137. Tagg SL, et al. 2-Methoxyoestradiol-3,17-O,O-bis-sulphamate and 2-deoxy-Dglucose in combination: a potential treatment for breast and prostate cancer. Br J Cancer. 2008;99(11):1842-1848.

138. Gang BP, Dilda PJ, Hogg PJ, Blackburn AC. Targeting of two aspects of metabolism in breast cancer treatment. Cancer Biol Ther. 2014;15(11):1533-1541.

139. Sutendra G, et al. Mitochondrial activation by inhibition of PDKII suppresses HIF1a signaling and angiogenesis in cancer. Oncogene. 2013;32(13):1638-1650.

140. Chu QS, et al. A phase I open-labeled, single-arm, dose-escalation, study of dichloroacetate (DCA) in patients with advanced solid tumors. Invest New Drugs. 2015;33(3):603-610.

141. Valvona CJ, Fillmore HL, Nunn PB, Pilkington GJ. The regulation and function of lactate dehydrogenase A: therapeutic potential in brain tumor. Brain Pathol. 2016;26(1):3-17.

142. Rai G, et al. Discovery and optimization of potent, cell-active pyrazole-based inhibitors of lactate dehydrogenase (LDH). J Med Chem. 2017;60(22):9184-9204.

143. Pivovarova AI, MacGregor GG. Glucose-dependent growth arrest of leukemia cells by MCT1 inhibition: feeding Warburg's sweet tooth and blocking acid export as an anticancer strategy. Biomed Pharmacother. 2018;98:173-179.

144.Benjamin D, et al. Dual inhibition of the lactate transporters MCT1 and MCT4 is synthetic lethal with metformin due to NAD+ depletion in cancer cells. Cell Rep. 2018;25(11):3047-3058.e4.

145. Payen VL, Mina E, Van Hée VF, Porporato PE, Sonveaux P. Monocarboxylate transporters in cancer. Mol Metab. 2020;33:48-66.

146. Hong CS, et al. MCT1 modulates cancer cell pyruvate export and growth of tumors that co-express MCT1 and MCT4. Cell Rep. 2016;14(7):1590-1601.

147. Lacroix R, Rozeman EA, Kreutz M, Renner K, Blank CU. Targeting tumor-associated acidity in cancer immunotherapy. Cancer Immunol Immunother. 2018;67(9):1331-1348.
148.Serganova I, et al. LDH-A regulates the tumor microenvironment via HIF-signaling and modulates the immune response. PLoS One. 2018;13(9):e0203965.

149. Beloueche-Babari M, et al. Monocarboxylate transporter 1 blockade with AZD3965 inhibits lipid biosynthesis and increases tumour immune cell infiltration. BrJCancer. 2020;122(6):895-903.

150. Chafe SC, et al. Targeting hypoxia-induced carbonic anhydrase IX enhances immune-checkpoint blockade locally and systemically. Cancer Immunol Res. 2019;7(7):1064-1078.

151. Demas DM, et al. Glutamine metabolism drives growth in advanced hormone receptor positive breast cancer. Front Oncol. 2019;9:686.

152. Schulte ML, et al. Pharmacological blockade of ASCT2-dependent glutamine transport leads to antitumor efficacy in preclinical models. Nat Med. 2018;24(2):194-202.

153. van Geldermalsen M, et al. Benzylserine inhibits breast cancer cell growth by disrupting intracellular amino acid homeostasis and triggering amino acid response pathways. BMC Cancer. 2018;18(1):689.

154.Lukey MJ, et al. Liver-type glutaminase GLS2 is a druggable metabolic node in luminal-subtype breast cancer. Cell Rep. 2019;29(1):76-88.e7.

155. Lampa M, et al. Glutaminase is essential for the growth of triple-negative breast cancer cells with a deregulated glutamine metabolism pathway and its suppression synergizes with $\mathrm{mTOR}$ inhibition. PLoS One. 2017;12(9):e0185092.

156. Fako VE, Wu X, Pflug B, Liu JY, Zhang JT. Repositioning proton pump inhibitors as anticancer drugs by targeting the thioesterase domain of human fatty acid synthase. JMed Chem. 2015;58(2):778-784.

157. Wang BY, et al. Intermittent high dose proton pump inhibitor enhances the antitumor effects of chemotherapy in metastatic breast cancer. JExp Clin Cancer Res. 2015;34:85.

158. Coll AP, et al. GDF15 mediates the effects of metformin on body weight and energy balance. Nature. 2020;578(7795):444-448.

159. Chung YC, Chang CM, Wei WC, Chang TW, Chang KJ, Chao WT. Metformin-induced caveolin-1 expression promotes T-DM1 drug efficacy in breast cancer cells. Sci Rep. 2018;8(1):3930.

160.Samuel SM, Varghese E, Kubatka P, Triggle CR, Büsselberg D. Metformin: the answer to cancer in a flower? Current knowledge and future prospects of metformin as an anti-cancer agent in breast cancer. Biomolecules. 2019;9(12):E846.

161. Lord SR, et al. Integrated pharmacodynamic analysis identifies two metabolic adaption pathways to metformin in breast cancer. Cell Metab. 2018;28(5):679-688.e4.

162. Quintela-Fandino M, et al. Randomized phase O/I trial of the mitochondrial inhibitor ME-344 or placebo added to bevacizumab in early HER2-negative breast cancer. Clin Cancer Res. 2020;26(1):35-45.

163. Cao J, et al. Decylubiquinone suppresses breast cancer growth and metastasis by inhibiting angiogenesis via the ROS/p53/ BAI1 signaling pathway. Angiogenesis. 2020;23(3):325-338.

164. Hojman P, Gehl J, Christensen JF, Pedersen BK. Molecular mechanisms linking exercise to 
cancer prevention and treatment. Cell Metab. 2018;27(1):10-21.

165. Klement RJ, Brehm N, Sweeney RA. Ketogenic diets in medical oncology: a systematic review with focus on clinical outcomes. Med Oncol. 2020;37(2):14.

166. Kanarek N, Petrova B, Sabatini DM. Dietary modifications for enhanced cancer therapy. Nature. 2020;579(7800):507-517.

167. Rieger J, et al. ERGO: a pilot study of ketogenic diet in recurrent glioblastoma. Int JOncol. 2014;44(6):1843-1852.

168. Cohen CW, et al. A ketogenic diet reduces central obesity and serum insulin in women with ovarian or endometrial cancer. J Nutr. 2018;148(8):1253-1260.

169. Rodrigues LM, Uribe-Lewis S, Madhu B, Honess DJ, Stubbs M, Griffiths JR. The action of $\beta$-hydroxybutyrate on the growth, metabolism and global histone $\mathrm{H} 3$ acetylation of spontaneous mouse mammary tumours: evidence of a $\beta$-hydroxybutyrate paradox. Cancer Metab. 2017;5:4

170. Nencioni A, Caffa I, Cortellino S, Longo VD. Fasting and cancer: molecular mechanisms and clinical application. Nat Rev Cancer. 2018;18(11):707-719.

171. de Groot S, et al. The effects of short-term fasting on tolerance to (neo) adjuvant chemotherapy in HER2-negative breast cancer patients: a randomized pilot study. BMC Cancer. 2015;15:652.

172. Antoniewicz MR. A guide to ${ }^{13} \mathrm{C}$ metabolic flux analysis for the cancer biologist. Exp Mol Med. 2018;50(4):19.

173. Maher EA, et al. Metabolism of [U-13 C]glucose in human brain tumors in vivo. NMR Biomed. 2012;25(11):1234-1244.

174. Sadeghzadeh M, et al. Development and radiosynthesis of the first ${ }^{18} \mathrm{~F}$-labeled inhibitor of monocarboxylate transporters (MCTs). J Labelled Comp Radiopharm. 2019;62(8):411-424.

175. Generali D, et al. Phosphorylated ERalpha, HIF-1alpha, and MAPK signaling as predictors of primary endocrine treatment response and resistance in patients with breast cancer. J Clin Oncol. 2009;27(2):227-234.

176. Helczynska K, et al. Hypoxia-inducible factor-2alpha correlates to distant recurrence and poor outcome in invasive breast cancer. Cancer Res. 2008;68(22):9212-9220.

177. Luo D, Liu H, Lin D, Lian K, Ren H. The clinicopathologic and prognostic value of hypoxia-inducible factor-2 $\alpha$ in cancer patients: a systematic review and meta-analysis. Cancer Epidemiol Biomarkers Prev. 2019;28(5):857-866.
178. Liu YH, et al. Diffuse optical spectroscopy for monitoring the responses of patients with breast cancer to neoadjuvant chemotherapy: a meta-analysis. Medicine (Baltimore). 2018;97(41):e12683.

179. Tromberg BJ, et al. Predicting responses to neoadjuvant chemotherapy in breast cancer: ACRIN 6691 trial of diffuse optical spectroscopic imaging. Cancer Res. 2016;76(20):5933-5944.

180. Cheng J, et al. 18F-fluoromisonidazole PET/CT: a potential tool for predicting primary endocrine therapy resistance in breast cancer. J Nucl Med. 2013;54(3):333-340.

181. Ho D, et al. Impact of serum HER2, TIMP-1, and CAIX on outcome for HER2+ metastatic breast cancer patients: CCTG MA.31 (lapatinib vs. trastuzumab). Breast Cancer Res Treat. 2017;164(3):571-580.

182.van Kuijk SJ, Yaromina A, Houben R, Niemans $\mathrm{R}$, Lambin P, Dubois LJ. Prognostic significance of carbonic anhydrase IX expression in cancer patients: a meta-analysis. Front Oncol. 2016;6:69.

183. Alves WEFM, et al. CAIX is a predictor of pathological complete response and is associated with higher survival in locally advanced breast cancer submitted to neoadjuvant chemotherapy. BMC Cancer. 2019;19(1):1173.

184.Garcia S, et al. Poor prognosis in breast carcinomas correlates with increased expression of targetable CD146 and c-Met and with proteomic basal-like phenotype. Hum Pathol. 2007;38(6):830-841.

185. Yu M, et al. The prognostic value of GLUT1 in cancers: a systematic review and meta-analysis. Oncotarget. 2017;8(26):43356-43367.

186.Coudert B, et al. Use of [(18)F]-FDG PET to predict response to neoadjuvant trastuzumab and docetaxel in patients with HER2-positive breast cancer, and addition of bevacizumab to neoadjuvant trastuzumab and docetaxel in [(18)F]-FDG PET-predicted non-responders (AVATAXHER): an open-label, randomised phase 2 trial. Lancet Oncol. 2014;15(13):1493-1502.

187. Humbert O, et al. Breast cancer blood flow and metabolism on dual-acquisition ${ }^{18} \mathrm{~F}$-FDG PET: correlation with tumor phenotype and neoadjuvant chemotherapy response. J Nucl Med. 2018;59(7):1035-1041.

188. Tian F, Shen G, Deng Y, Diao W, Jia Z. The accuracy of ${ }^{18}$ F-FDG PET/CT in predicting the pathological response to neoadjuvant chemotherapy in patients with breast cancer: a meta-analysis and systematic review. Eur Radiol.2017;27(11):4786-4796.

189.Wang F, et al. Predictive role of the overexpression for CXCR4, C-Met, and VEGF-C among breast cancer patients: a meta-analysis. Breast. 2016;28:45-53.

190. Nieto Y, et al. Prognostic analysis of tumour angiogenesis, determined by microvessel density and expression of vascular endothelial growth factor, in high-risk primary breast cancer patients treated with high-dose chemotherapy. $\mathrm{Br} \mathrm{JCan-}$ cer. 2007;97(3):391-397.

191. Goussia A, et al. Associations of angiogenesis-related proteins with specific prognostic factors, breast cancer subtypes and survival outcome in early-stage breast cancer patients. A Hellenic Cooperative Oncology Group (HeCOG) trial. PLoS One. 2018;13(7):e0200302.

192.Zhang Z, Luo G, Tang H, Cheng C, Wang P. Prognostic significance of high VEGF-C expression for patients with breast cancer: an update meta analysis. PLoS One. 2016;11(11):e0165725.

193. Li Q, et al. Low-dose anti-angiogenic therapy sensitizes breast cancer to PD-1 blockade. Clin Cancer Res. 2020;26(7):1712-1724.

194. Wu FTH, et al. Pre- and post-operative anti-PD-L1 plus anti-angiogenic therapies in mouse breast or renal cancer models of micro- or macro-metastatic disease. Br JCancer 2019;120(2):196-206.

195.Schmittnaegel M, et al. Dual angiopoietin-2 and VEGFA inhibition elicits antitumor immunity that is enhanced by PD-1 checkpoint blockade. Sci Transl Med. 2017;9(385):eaak9670.

196. Allen E, et al. Combined antiangiogenic and anti-PD-L1 therapy stimulates tumor immunity through HEV formation. Sci Transl Med. 2017;9(385):eaak9679.

197. Tian L, et al. Mutual regulation of tumour vessel normalization and immunostimulatory reprogramming. Nature. 2017;544(7649):250-254.

198. Hlouschek J, Ritter V, Wirsdörfer F, Klein D, Jendrossek V, Matschke J. Targeting SLC25A10 alleviates improved antioxidant capacity and associated radioresistance of cancer cells induced by chroniccycling hypoxia. Cancer Lett. 2018;439:24-38.

199.Wang H, Jiang H, Van De Gucht M, De Ridder M. Hypoxic radioresistance: can ROS be the key to overcome it? Cancers (Basel). 2019;11(1):E112.

200.Indira Chandran V, et al. Hypoxia attenuates trastuzumab uptake and trastuzumab-emtansine (T-DM1) cytotoxicity through redistribution of phosphorylated caveolin-1. Mol Cancer Res. 2020;18(4):644-656. 